\title{
Sliding contact conditions using the master slave approach with application on geometrically non-linear beams
}

\author{
José J. Muñoz ${ }^{1}$, Gordan Jelenić ${ }^{*, 1}$ \\ Department of Aeronautics, Imperial College London, UK
}

In memory of Mike Crisfield and the years spent working together

\begin{abstract}
Frictionless sliding conditions between two bodies are usually defined using either the method of Lagrangian multipliers or by prescribing an artificial (penalty) stiffness which resists the penetration at the contact point. Both of these methods impose the condition that the contact force should be normal to the contact surface, with the Lagrangian multiplier or the penalty parameter serving as a measure of this force. In this work, an alternative approach is undertaken: the frictionless sliding condition is defined through a relationship between nodal parameters of the virtual displacements of a discretised principle of virtual work. This method, which does not involve additional force parameters or degrees of freedom, is known as the master slave or the minimum set method and is particularly con venient for displacement based finite element implementation. The method is analysed in detail in context of bilateral sliding constraints characteristic of prismatic and cylindrical joints in flexible beam assemblies undergoing large overall motion. Two numerical examples are presented and assessed against the results in the literature.
\end{abstract}

Keywords: Master slave method; Contact; Sliding; Large rotations; Joints; Beams

\section{Motivation and introduction}

The present work is motivated by the authors' desire to model translational joints (prismatic and cylindrical) in various types of flexible mechanisms using the master slave approach (Crisfield, 1997; Crisfield and Jelenić, 1998; Crisfield and Jelenić, 2000; Ibrahimbegović and Mamouri, 2000; Jelenić and Crisfield, 1996, 2001; Mitsugi, 1997; Muñoz et al., 2003) in a kinematically rigorous way. As given in these references, the method only relates the original nodal degrees of freedom (the slave degrees of freedom) of a

\footnotetext{
* Corresponding author. Address: Department of Civil Engineering, University of Rijeka, Viktora Cara Emina 5, 51000 Rijeka Fiume, Croatia. Tel.: +385 51352 114; fax: +385 51332816 .

Email addresses: jose.munoz@imperial.ac.uk (J.J. Munoz), gordan@gradri.hr (G. Jelenić).

${ }^{1}$ Financially supported by the Engineering and Physical Sciences Research Council under grants GR/R 04171/01 and AF/100089.
} 
finite element to an extended set of degrees of freedom, which are again specific to that node. This extended set includes the released degrees of freedom defined by the joint which is being modelled and the global, master, degrees of freedom, which are shared by all the other elements which join at that node. For joints which cannot be defined with respect to a single material point, such an approach is only kinematically approximate.

By considering, for example, a prismatic joint, which is initially at the position of a node in the middle of the horizontal beam shown in Fig. 1a, this procedure will invariably result in the direction of the released degree of freedom in a deformed state being defined by the tangent to the beam at that node. This, as can be seen from Fig. 1b and c, is clearly a kinematically inconsistent result. This anomaly has already drawn certain amount of attention in the literature (Bauchau, 2000; Bauchau and Bottasso, 2001), where it has been rectified by a proper definition of the sliding contact condition using the method of Lagrangian multipliers. The authors named such a joint (Fig. 2) the sliding joint, and in this paper we will adopt this terminology to describe what we earlier referred to as the "kinematically rigorous translational joint". More on the method of Lagrangian multipliers as applied to the problems of multi-body dynamics may also be found in García de Jalón and Bayo (1994), Géradin and Cardona (2001) and von Schwerin (1999), among many other sources.

Here, we attempt to formulate the sliding joint without resorting to Lagrangian multipliers or the penalty method. This choice is motivated by the presence of standard complexities associated with these techniques. In particular, using the Lagrangian multipliers creates an additional degree of freedom (the multiplier) for each constraint these are the force degrees of freedom and, as such, give rise to the problems of well-posedness of the system of equations with mixed unknowns. Furthermore, the matrix of coefficients of the mixed system is not positive definite even in a stable region, which makes the occurrence of divergence-type instabilities less obvious. In dynamic problems, we are also left to deal with the coupled system of differential and algebraic equations, which require specially designed solution procedures. On the other hand, when the constraint condition is enforced through a penalty function there cannot exist the problems given above since the parameter is not an unknown, but this method leads to a new problem: the penalty method is inherently approximate, with its accuracy directly related to the magnitude of the chosen penalty parameter. By modifying this parameter the accuracy can be improved, but this is done at the expense of incurring the numerical problems due to the increased ill-conditioning

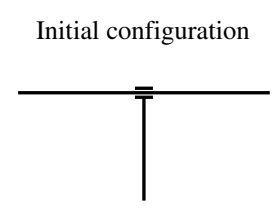

(a)

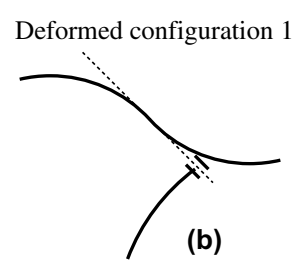

Fig. 1. Prismatic joint.

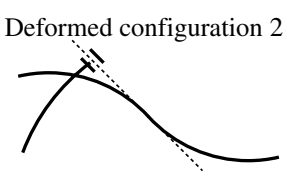

(c)

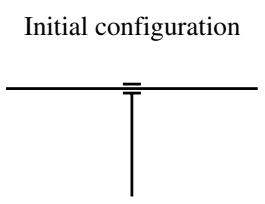

Deformed configuration 1

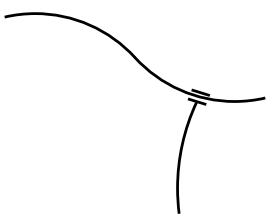

Fig. 2. Sliding joint.
Deformed configuration 2

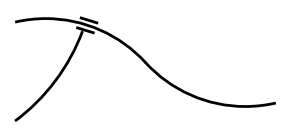


of the system. The so-called augmented Lagrange method (e.g. Géradin and Cardona, 2001) combines the advantages of the two approaches in order to remove the approximate character of the penalty method, while retaining its advantage of providing a positive definite Hessian at a stable equilibrium for a suitably chosen penalty parameter. In a similar vein, another constant scaling may be introduced to eliminate the problem of different orders of magnitude for the unknown degrees of freedom. The augmented Lagrangian method thus eliminates most of the above problems at the expense of having to deal with the choice of two sets of problem-dependent constant parameters. The Lagrangian multipliers, however, always remain the global degrees of freedom to be added to the original set of the kinematic degrees of freedom.

The master slave method deals with the minimum set of the kinematic degrees of freedom and in this sense cannot suffer from the difficulties mentioned above. The purpose of this paper is to retain this principal feature of the method, and at the same time generalise its node-based concepts to allow the modelling of sliding joints along a deformable surface. A very related approach has recently been undertaken (Marjamäki and Mäkinen, 2003) in order to analyse the sliding of a spring along a deformable planar beam.

Although our principal motivation is to obtain a realistic model of the sliding joints in $3 \mathrm{D}$ beams, in Section 2 the theory will first be derived by considering a genuine point-on-surface contact between 3D continua. It should be noted, however, that here we do not address the implications of the method to contact mechanics, although we believe that such implications exist. In particular, throughout the paper we limit our attention to bilateral contacts only, i.e. we do not address the issues of contact detection and we assume the contact force to be of either direction. In Section 3, we apply the theory to the problem of sliding joints in $3 \mathrm{D}$ beams, where the justification for the assumption of bilateral contact becomes obvious. Section 4 presents two relevant numerical examples.

\section{Point-on-surface sliding contact in 3D elastodynamics}

We consider a simple system of two elastic bodies which are in contact with one another through a single point. The kinematics of the contact is analysed first in order to establish its implications on the nature of the virtual displacements leading to the governing equations of motion. A suitable finite-element discretisation is performed next, which takes account of this contact condition, eventually leading to a system of equations with the minimum number of degrees of freedom.

The two bodies are denoted as $\mathscr{B}^{A}$ and $\mathscr{B}^{B}$ and are represented by a smooth and invertible mapping into the ambient space $\phi: \mathscr{B}^{I} \rightarrow \mathbb{R}^{3}, I=A, B$ as shown in Fig. 3. Let the surfaces of the bodies $\partial \mathscr{B}^{A}$ and $\partial \mathscr{B}^{B}$ be Lipshitz-continuous and consist of two parts each: the parts $\mathscr{E}_{u}^{A}$ and $\mathscr{E}_{u}^{B}$ with prescribed kinematics and the parts $\mathscr{E}_{p}^{A}$ and $\mathscr{E}_{p}^{B B}$ with a prescribed surface traction such that

$$
\mathscr{E}_{u}^{I} \cap \mathscr{E}_{p}^{I}=\emptyset \quad \text { and } \quad \mathscr{E}_{u}^{I} \cup \mathscr{E}_{p}^{I}=\partial \mathscr{B}^{I} \quad I=A, B
$$

The distinction between the mappings that take place at different times will be made by defining the mapping $\phi_{t}: \mathbb{R}^{3} \times \mathbb{R}_{+} \rightarrow \mathbb{R}^{3}$. At time $t, \mathscr{B}^{I}, \mathscr{E}_{u}$ and $\mathscr{E}_{p}^{I}$ are mapped into $\phi_{t}\left(\mathscr{B}^{I}\right) \subset \mathbb{R}^{3}, \phi_{t}\left(\mathscr{E}_{u}\right) \subset \mathbb{R}^{2}$ and $\phi_{t}\left(\mathscr{E}_{p}\right) \subset \mathbb{R}^{2}$, where $\phi_{t}(\bullet)$ is a shorthand notation for $\phi(\bullet, t)$. Also, for a given material particle $P \in \mathscr{B}$, let us also define the mapping $\chi: \mathscr{B} \rightarrow \mathbb{R}^{3}$, and the reference and deformed position vectors at time $t$ as $\mathbf{X}_{P}=\chi(P)$ and $\boldsymbol{x}_{P}=\phi_{t}(P)$, respectively.

In the deformed configuration, the contact between $\mathscr{B}^{A}$ and $\mathscr{B}^{B}$ is established at some material points $A_{1}$ and $B_{1}$, i.e.

$$
\boldsymbol{x}_{A_{1}}=\boldsymbol{x}_{B_{1}},
$$

as also shown in Fig. 3. 


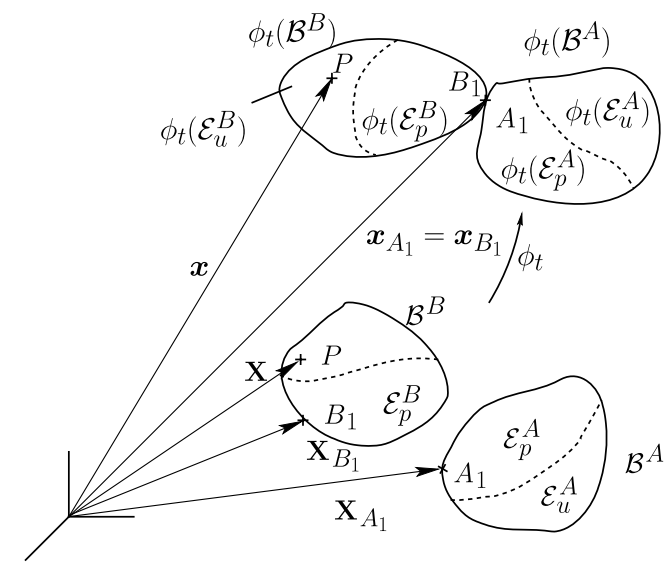

Fig. 3. Mappings between the reference configuration and the deformed configuration.

The present formulation is based on the following hypotheses:

H1 The material point $A_{1}$ on body $\mathscr{B}^{A}$ remains in contact with body $\mathscr{B}^{B}$ throughout the motion, whereas material point $B_{1}$ does not have to stay in contact with $\mathscr{B}^{A}$, i.e. point $A_{1}$ slides over the surface of $\mathscr{B}^{B}$.

H2 At time $t$, body $\mathscr{B}^{A}$ exerts a force on point $B_{1}$ of body $\mathscr{B}^{B}$ denoted $\mathbf{S}_{B_{1}}$, which is taken to be equal in magnitude and opposite in the direction of the force $\mathbf{S}_{A_{1}}$, exerted on point $A_{1}$ of body $\mathscr{B}^{A}$ by body $\mathscr{B}^{B}$, i.e.

$$
\mathbf{S}_{A_{1}}=-\mathbf{S}_{B_{1}} \text {. }
$$

This assumption is motivated by Newton's third law, valid for the contact forces between mass particles. H3 Only frictionless contact is considered, which implies that the interaction forces between the bodies point in the negative directions of the normals $\boldsymbol{n}_{A_{1}}$ and $\boldsymbol{n}_{B_{1}}$ to the surfaces $\partial \mathscr{B}^{A}$ and $\partial \mathscr{B}^{B}$, i.e.

$$
\mathbf{S}_{A_{1}}=-\left\|\mathbf{S}_{A_{1}}\right\| \boldsymbol{n}_{A_{1}} \text { and } \mathbf{S}_{B_{1}}=-\left\|\mathbf{S}_{B_{1}}\right\| \boldsymbol{n}_{B_{1}} .
$$

Note that the normals are uniquely defined since the surfaces are Lipschitz-continuous.

\subsection{Cauchy's equation of motion and its weak form}

The (local) Cauchy's equation of motion is defined in the deformed configuration as (Marsden and Hughes, 1994):

$$
\operatorname{div} \boldsymbol{\sigma}+\mathbf{b}=\rho \ddot{\boldsymbol{x}}
$$

on $\phi_{t}\left(\mathscr{B}^{A} \cup \mathscr{B}^{B}\right)$, where $\boldsymbol{\sigma}$ is the Cauchy (true) stress tensor, $\operatorname{div} \boldsymbol{\sigma}=\boldsymbol{\sigma} \nabla_{\boldsymbol{x}}$, b is a distributed loading per unit volume, $\rho$ is the current density of the material and here and throughout the paper $(\bullet)$ and $(\bullet)$ indicate material first and second time differentiation. This equation has to be complemented with the natural boundary conditions

$$
\begin{aligned}
& \boldsymbol{\sigma n}-\mathbf{s}=\mathbf{0} \quad \text { on } \quad \phi_{t}\left(\mathscr{E}_{p}^{A} \cup \mathscr{E}_{p}^{B}\right) \backslash\left(A_{1} \cup B_{1}\right), \\
& \lim _{\mathscr{I}_{I_{1}} \rightarrow 0} \int_{\mathscr{A}_{I_{1}}} \boldsymbol{\sigma} \mathrm{d} S \boldsymbol{n}+\mathbf{S}_{I_{1}}=\mathbf{0} \quad \text { at } I_{1}, \quad I=A, B,
\end{aligned}
$$


where $\boldsymbol{n}$ is the outward normal to surface $\phi_{t}\left(\mathscr{E}_{p}\right)$ and $\mathbf{s}$ is a distributed loading per unit area of this surface, while $\mathscr{A}_{A_{1}} \subset \phi_{t}\left(\mathscr{E}_{p}^{A}\right)$ and $\mathscr{A}_{B_{1}} \subset \phi_{t}\left(\mathscr{E}_{p}^{B}\right)$ denote the surface areas in the neighbourhoods of $A_{1}$ and $B_{1}$ respectively at time $t$. Furthermore, the essential boundary condition reads

$$
\boldsymbol{x}=\boldsymbol{x} \quad \text { on } \phi_{t}\left(\mathscr{E}_{u}^{I}\right) \quad I=A, B,
$$

where $\boldsymbol{x}$ is a prescribed displacement field. The weak form of Cauchy's equation of motion (the virtual work) is obtained by taking the dot product of Eqs. (1) (3) with the admissible variations $\delta \boldsymbol{x}$ of the deformed configuration (which are therefore required to vanish on $\phi_{t}\left(\mathscr{E}_{u}\right)$ thus satisfying Eq. (4)). Note that by defining a displacement of a material point as $\boldsymbol{p}=\boldsymbol{x}_{t}-\boldsymbol{x}_{0}$, the admissible variation $\delta \boldsymbol{x}$ of the deformed configuration may be substituted by the virtual displacement $\delta p$. By integrating the dot product of Eqs. (1) (3) with $\delta \boldsymbol{p}$ over the domain $\phi_{t}\left(\mathscr{B}^{A}\right) \cup \phi_{t}\left(\mathscr{B}^{B}\right)$, we obtain the following weak form,

$$
\begin{aligned}
G(\boldsymbol{x}, \delta \boldsymbol{p}) \equiv & \sum_{I}\left[\int_{\phi_{t}\left(\mathscr{B}^{I}\right)} \delta \boldsymbol{p} \cdot(\operatorname{div} \boldsymbol{\sigma}+\mathbf{b}-\rho \ddot{\boldsymbol{x}}) \mathrm{d} V-\int_{\phi_{t}\left(\mathscr{E}_{p}^{I} \backslash I_{1}\right)} \delta \boldsymbol{p} \cdot(\boldsymbol{\sigma} \boldsymbol{n}-\mathbf{s}) \mathrm{d} S\right. \\
& \left.-\delta \boldsymbol{p}_{I_{1}} \cdot\left(\lim _{\mathscr{A}_{I_{1}} \rightarrow 0} \int_{\mathscr{A}^{I}} \boldsymbol{\sigma} \mathrm{d} S \boldsymbol{n}+\mathbf{S}_{I_{1}}\right)\right]=0 .
\end{aligned}
$$

Introducing the identity $\delta \boldsymbol{p} \cdot \operatorname{div} \boldsymbol{\sigma}=\operatorname{div}(\boldsymbol{\sigma} \delta \boldsymbol{p})-\operatorname{grad} \delta \boldsymbol{p}: \boldsymbol{\sigma}$, where $\operatorname{grad} \delta \boldsymbol{x}=\nabla_{\boldsymbol{x}} \delta \boldsymbol{x}$, a colon indicates a double tensor contraction, and applying the divergence theorem via $\int_{\phi_{t}(\mathscr{B})} \operatorname{div}(\boldsymbol{\sigma} \delta \boldsymbol{p}) \mathrm{d} V=\int_{\partial \phi_{t}(\mathscr{B})} \delta \boldsymbol{p} \cdot \boldsymbol{\sigma n} \mathrm{d} S$ and noting $\int_{\phi_{t}\left(\mathscr{E}_{u}^{I}\right)} \delta \boldsymbol{p} \cdot \boldsymbol{\sigma} \boldsymbol{n} \mathrm{d} S=0, I=A, B$ due to the kinematic admissibility of $\delta \boldsymbol{p}$, the previous equation turns into

$$
G(\boldsymbol{x}, \delta \boldsymbol{p}) \equiv \sum_{I}\left\{\int_{A, B}\left[\nabla_{\phi_{t}\left(\mathscr{B}^{I}\right)} \delta \boldsymbol{x}: \boldsymbol{\sigma}+\delta \boldsymbol{p} \cdot(\rho \ddot{\boldsymbol{x}}-\mathbf{b})\right] \mathrm{d} V-\int_{\phi_{t}\left(\mathscr{E}_{p}^{I}\right)} \delta \boldsymbol{p} \cdot \mathbf{s} \mathrm{d} S+\delta \boldsymbol{p}_{I_{1}} \cdot \mathbf{S}_{I_{1}}\right\}=0,
$$

where we have changed the sign for convenience. In order to express the weak form in (5) as integrals over the reference domains, we introduce first the Piola transform of $\boldsymbol{\sigma}$ via $\boldsymbol{\sigma}=J^{-1} \mathbf{P F}^{\mathrm{T}}$ with $J=\operatorname{det} \mathbf{F}$, $\mathbf{F}=$ GRAD $\boldsymbol{x}=\nabla_{\mathbf{X}} \boldsymbol{x}$ and $\mathbf{P}$ the first Piola Kirchhoff stress tensor (Marsden and Hughes, 1994). By inserting this transformation, evaluating the tensor contraction via $\mathbf{A}: \mathbf{B}=\operatorname{tr}\left(\mathbf{A B}^{\mathrm{T}}\right)$ and using the identity $\left(\nabla_{\boldsymbol{x}} \delta \boldsymbol{p}\right) \mathbf{F}=\nabla_{\mathbf{X}} \delta \boldsymbol{p}$, the first term in (5) transforms into

$$
\int_{\phi_{t}(\mathscr{B})} \nabla_{\boldsymbol{p}} \delta \boldsymbol{x}: \boldsymbol{\sigma} \mathrm{d} V=\int_{\mathscr{B}} \operatorname{tr}\left[\left(\nabla_{\mathbf{X}} \delta \boldsymbol{p}\right) \mathbf{P}^{\mathrm{T}}\right] \mathrm{d} V_{0}=\int_{\mathscr{B}} \nabla_{\mathbf{X}} \delta \boldsymbol{p}: \mathbf{P} \mathrm{d} V_{0},
$$

where $V_{0}$ is the volume of $\mathscr{B}$ in the reference configuration. By making use of the conservation of mass $\rho \mathrm{d} V=\rho_{0} \mathrm{~d} V_{0}$, where $\rho_{0}$ is the density in the reference configuration, Nanson's formula $\boldsymbol{n} \mathrm{d} S=J \boldsymbol{F}^{-\mathrm{T}} \boldsymbol{n}_{0} \mathrm{~d} S_{0}$ (Ogden, 1984) and introducing Eq. (6), we finally obtain the following expression for the weak form:

$$
G(\boldsymbol{x}, \delta \boldsymbol{p}) \equiv \sum_{I}\left\{\int_{A, B}\left[\nabla_{\mathbf{X}} \delta \boldsymbol{p}: \mathbf{P}+\delta \boldsymbol{p} \cdot\left(\rho_{0} \ddot{\boldsymbol{x}}-\mathbf{b}_{0}\right)\right] \mathrm{d} V_{0}-\int_{\mathscr{E}_{p}^{I}} \delta \boldsymbol{p} \cdot \mathbf{s}_{0} \mathrm{~d} S_{0}+\delta \boldsymbol{p}_{I_{1}} \cdot \mathbf{S}_{I_{1}}\right\}=0,
$$

with $\mathbf{b}_{0}$ and $\mathbf{s}_{0}$ being the distributed loads per units of reference volume and area, respectively.

\subsection{Infinitesimal kinematic conditions and master slave approach}

We will now deduce a relation between the virtual displacements $\delta \boldsymbol{p}_{A_{1}}$ and $\delta \boldsymbol{p}_{B_{1}}$ at the contact points. Let us perturb the deformed configuration in Fig. 3 with an infinitesimal variation represented by the vector field $\epsilon \delta \boldsymbol{p}$, where $\epsilon$ is an arbitrarily small scalar. 
Following assumption $\mathrm{H} 1$ introduced at the beginning of the section, contact point $A_{1}$ at body $\mathscr{B}^{A}$ in such a perturbed state remains in contact with body $\mathscr{B}^{B}$, but this point is now in contact with a point $B_{2}$ at body $\mathscr{B}^{B}$, which is in general different from the contact point $B_{1}$ in the unperturbed deformed state. Fig. 4 shows this situation where the perturbed configuration has been denoted by $\phi_{t, \epsilon}$. According to this figure, the positions of the new contact points are given by:

$$
\begin{aligned}
& \boldsymbol{x}_{\epsilon}\left(\mathbf{X}_{B_{2}}\right)=\boldsymbol{x}\left(\mathbf{X}_{B_{2}}\right)+\epsilon \delta p\left(\mathbf{X}_{B_{2}}\right)=\boldsymbol{x}\left(\mathbf{X}_{B_{1}}+\epsilon \delta \mathbf{T}\right)+\epsilon \delta \boldsymbol{p}\left(\mathbf{X}_{B_{1}}+\epsilon \delta \mathbf{T}\right), \\
& \boldsymbol{x}_{\epsilon}\left(\mathbf{X}_{A_{1}}\right)=\boldsymbol{x}\left(\mathbf{X}_{A_{1}}\right)+\epsilon \delta \boldsymbol{p}\left(\mathbf{X}_{A_{1}}\right),
\end{aligned}
$$

where $\epsilon \delta \mathbf{T}$ is the difference in the reference configuration of the position vectors of $\mathbf{X}_{B_{2}}$ and $\mathbf{X}_{B_{1}}$ (see Fig. 5), i.e.

$$
\mathbf{X}_{B_{2}}=\mathbf{X}_{B_{1}}+\epsilon \delta \mathbf{T} \text {. }
$$

The contact condition at the perturbed deformed state is, of course,

$$
\boldsymbol{x}_{\epsilon}\left(\mathbf{X}_{B_{2}}\right)=\boldsymbol{x}_{\epsilon}\left(\mathbf{X}_{A_{1}}\right) \text {. }
$$

Substituting (8) into this result, computing its derivative with respect to $\epsilon$ and setting $\epsilon=0$ gives

$$
\left.\delta p\left(\mathbf{X}_{A_{1}}\right) \equiv \frac{\mathrm{d}}{\mathrm{d} \epsilon}\right|_{\epsilon} \boldsymbol{x}_{\epsilon}\left(\mathbf{X}_{A_{1}}\right)=\left.\frac{\mathrm{d}}{\mathrm{d} \epsilon}\right|_{\epsilon} \boldsymbol{x}_{\epsilon}\left(\mathbf{X}_{B_{2}}\right)=\left.\boldsymbol{F}\right|_{B_{1}} \delta \mathbf{T}+\delta \boldsymbol{p}\left(\mathbf{X}_{B_{1}}\right)=\delta \boldsymbol{t}_{B_{1}}+\delta \boldsymbol{p}\left(\mathbf{X}_{B_{1}}\right),
$$

with $\left.\boldsymbol{F}\right|_{B_{1}}=\left.\nabla_{\mathbf{X}} \boldsymbol{x}\right|_{B_{1}}=$ GRAD $\left.\boldsymbol{x}\right|_{B_{1}}$ being the deformation gradient at $B_{1}$ and the tangent to the deformed surface at $B_{1}, \delta t_{B_{1}}=\left.\boldsymbol{F}\right|_{B_{1}} \delta \mathbf{T}$, the push-forward of the material tangent vector $\delta \mathbf{T}$ at point $B_{1}$. By para-

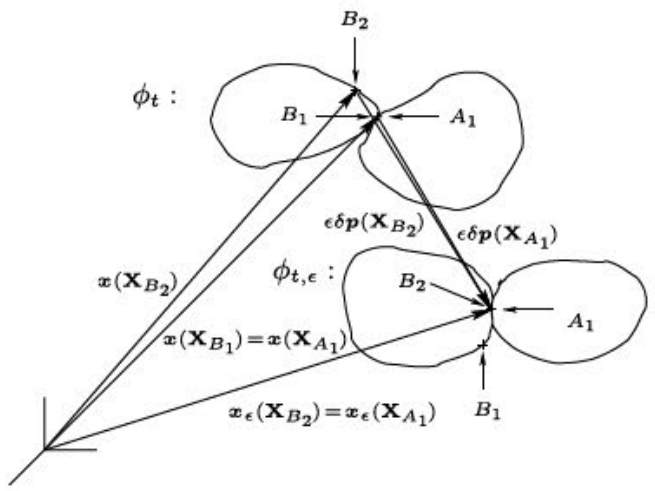

Fig. 4. Deformed and perturbed configuration.

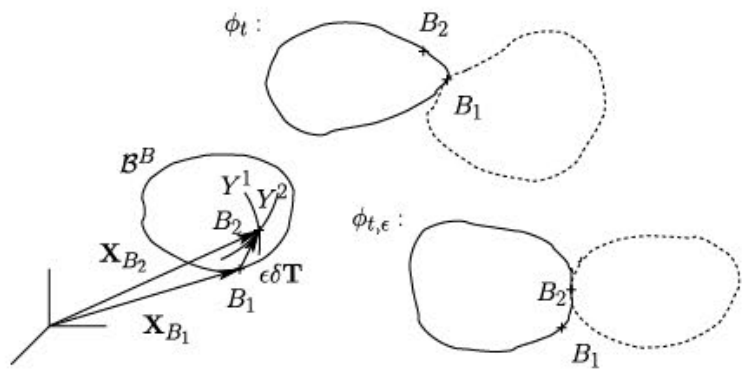

Fig. 5. Contact points on body $\mathscr{B}^{B}$. 
metrising the boundary surface $\partial \mathscr{B}^{B}$ using the coordinate chart $\mathbf{Y}=\left\{Y^{1}, Y^{2}\right\}$, this vector can be expressed as

$$
\delta \mathbf{T}=\left.\frac{\mathrm{d}}{\mathrm{d} \epsilon}\right|_{\epsilon} \mathbf{X}\left(Y^{1}+\epsilon \delta Y^{1}, Y^{2}+\epsilon \delta Y^{2}\right)=\left.\frac{\partial \mathbf{X}}{\partial Y^{1}}\right|_{B_{1}} \delta Y^{1}+\left.\frac{\partial \mathbf{X}}{\partial Y^{2}}\right|_{B_{1}} \delta Y^{2}=\left.\mathbf{F}_{Y_{1}}\right|_{B_{1}} \delta \mathbf{Y}
$$

with $\delta \mathbf{Y}=\left\{\delta Y^{1}, \delta Y^{2}\right\}$ and $\mathbf{F}_{Y}=\nabla_{\mathbf{Y}} \mathbf{X}$. This allows us to write $\delta \mathbf{t}_{B_{1}}$ as

$$
\delta \mathbf{t}_{B_{1}}=\mathbf{F}_{B_{1}} \delta \mathbf{Y}
$$

where $\mathbf{F}_{B_{1}}=\left.\left.\mathbf{F}\right|_{B_{1}} \mathbf{F}_{Y}\right|_{B_{1}}$.

Replacing $\delta \mathbf{p}_{A_{1}}=\delta \mathbf{p}\left(\mathbf{X}_{A_{1}}\right)$ in (7) with the expression in the right-hand side of Eq. (9), and recalling hypothesis $\mathrm{H} 2$ we obtain

$$
G(\mathbf{x}, \delta \mathbf{p}) \equiv \sum_{I}\left\{\int_{A, B}\left[\nabla_{\mathbf{X}} \delta \mathbf{p}: \mathbf{P}+\delta \mathbf{p} \cdot\left(\rho_{0} \ddot{\mathbf{x}}-\mathbf{b}_{0}\right)\right] \mathrm{d} V_{0}-\int_{\mathscr{E}_{p}^{I}} \delta \mathbf{p} \cdot \mathbf{s}_{0} \mathrm{~d} S_{0}\right\}-\delta \mathbf{t}_{B_{1}} \cdot \mathbf{S}_{B_{1}}=0,
$$

which, due to hypothesis H3 $\left(\mathbf{S}_{B_{1}}\right.$ is normal to surface $\partial \mathscr{B}^{B}$ at point $B_{1}$ and $\left.\delta \mathbf{t}_{B_{1}} \cdot \mathbf{n}_{B_{1}}=0\right)$, gives rise to the standard (and anticipated) result

$$
G(\mathbf{x}, \delta \mathbf{p}) \equiv \sum_{I}\left\{\int_{A, B}\left[\nabla_{\mathbf{X}} \delta \mathbf{p}: \mathbf{P}+\delta \mathbf{p} \cdot\left(\rho_{0} \ddot{\mathbf{x}}-\mathbf{b}_{0}\right)\right] \mathrm{d} V_{0}-\int_{\mathscr{E}_{p}^{I}} \delta \mathbf{p} \cdot \mathbf{s}_{0} \mathrm{~d} S_{0}\right\}=0 .
$$

The resulting weak form is therefore the same as the one obtained by considering the two bodies separately and without taking into account the sliding condition. This condition, however, remains implicit in the definition of the virtual displacement $\delta \mathbf{p}$ at the contact point.

\subsection{Finite element interpolation of the test functions}

Let us spatially discretise the problem by choosing $N^{I}$ nodal points on body $\mathscr{B}^{I}$ and approximating the virtual displacements $\delta \mathbf{p}$ by means of the Lagrangian polynomials $I^{j}(\mathbf{X})$ via

$$
\delta \mathbf{p}(\mathbf{X}) \doteq \delta \mathbf{p}^{h}(\mathbf{X})=I^{j}(\mathbf{X}) \delta \mathbf{p}_{j} \quad j=1, \ldots, N^{I},
$$

where summation over repeated indices is implied and the interpolation functions $I^{j}(\mathbf{X})$ satisfy the standard completeness conditions

$$
I^{j}\left(\mathbf{X}_{k}\right)=\delta_{k}^{j} \quad \sum_{j 1}^{N^{I}} I^{j}(\mathbf{X})=1 \quad \sum_{j 1}^{N^{I}} I^{\prime j}(\mathbf{X})=0,
$$

with $\delta_{k}^{j}$ being the Kronecker symbol.

In the beginning of this section it was assumed that the contact point $A_{1}$ on $\mathscr{B}^{A}$ was permanently in contact with body $\mathscr{B}^{B}$. Throughout this section we will consider the case in which each body is represented by a single finite element, denoted as $A$ and $B$ and having $N_{A}$ and $N_{B}$ nodes respectively. In this way, we will consider first the case where the sliding segment is fully contained within a single element, and only later analyse the modifications needed in order to include sliding along a set of finite elements.

In order to alleviate the notation in the subsequent sections, we will also assume that the contact node on element $A$ corresponds to node number $N_{A}$ (the last node according to the local numbering of nodes in the element).

Keeping these assumptions in mind, and noting the identity

$$
\left(\nabla_{\mathbf{X}} \delta \mathbf{p}^{h}\right): \mathbf{P}=\operatorname{tr}\left[\left(\delta \mathbf{p}_{j} \otimes \nabla_{\mathbf{X}} I^{j}\right) \mathbf{P}^{\mathrm{T}}\right]=\operatorname{tr}\left[\left(\delta \mathbf{p}_{j}\right) \otimes\left(\mathbf{P} \nabla_{\mathbf{X}} I^{j}\right)\right]=\delta \mathbf{p}_{j} \cdot \mathbf{P} \nabla I^{j}(\mathbf{X})
$$


the weak form in (10) can now be written in the following approximated form

$$
G^{h}\left(\mathbf{x}, \delta \mathbf{p}^{h}\right)=\delta \mathbf{p}_{j}^{A} \cdot \mathbf{g}^{A, j}+\delta \mathbf{p}_{j}^{B} \cdot \mathbf{g}^{B, j}=0
$$

where $\mathbf{g}^{I, j}=\mathbf{q}_{k}^{I, j}+\mathbf{q}_{d}^{I, j}-\mathbf{q}_{e}^{I, j}$ is the standard dynamic residual with

$$
\begin{aligned}
\mathbf{q}_{k}^{I, j} & =\int_{\mathscr{B}^{I}} \mathbf{P} \nabla I^{j}(\mathbf{X}) \mathrm{d} V_{0}, \\
\mathbf{q}_{d}^{I, j} & =\int_{\mathscr{B}^{I}} I^{j}(\mathbf{X}) \rho_{0} \ddot{\mathbf{x}} \mathrm{d} V_{0}, \\
\mathbf{q}_{e}^{I, j} & =\int_{\mathscr{B}^{I}} I^{j}(\mathbf{X}) \mathbf{b}_{0} \mathrm{~d} V_{0}+\int_{\mathscr{E}_{p}^{I}} I^{j}(\mathbf{X}) \mathbf{s}_{0} \mathrm{~d} S_{0}
\end{aligned}
$$

as the standard nodal vectors of internal, inertial and external forces at node $j$ of body $I$, respectively.

The interpolated form of relationship (9) follows from (11) as

$$
\delta \mathbf{p}_{N_{A}}^{A}=\delta \mathbf{t}_{B_{1}}+I_{B_{1}}^{j} \delta \mathbf{p}_{j}^{B},
$$

where $I_{B_{1}}^{j}=I^{j}\left(\mathbf{X}_{B_{1}}\right)$ and $\delta \mathbf{p}_{N_{A}}^{A}=\delta \mathbf{p}\left(\mathbf{X}_{A_{1}}\right)$. Eq. (13) provides the necessary sliding condition to develop the finite-element formulation using the master slave approach.

In order to put the problem into the required context, we will call the vector $\delta \mathbf{p}_{N_{A}}^{A}$ the virtual slave displacement, and all the vectors $\delta \mathbf{p}_{j}^{B}, j=1, \ldots, N_{B}$ the virtual master displacements. By calling the infinitesimal coordinate changes $\delta \mathbf{Y}$ the virtual released displacement, it becomes obvious that (13) gives the slave virtual displacement (of node $N_{A}$ in this example) as a function of this released displacement and master virtual displacements (of all the nodes on element $B$ ). Such a relationship is fundamental in the master slave approach (Jelenić and Crisfield, 1996; Jelenić and Crisfield, 2001), but we emphasise that in contrast to the results in these references, the present relationship is not confined to a single node. Instead, it involves a set of master nodes, which reflects the fact that the sliding condition takes into account deformability of the surface. The same basic approach was used in (Marjamäki and Mäkinen, 2003) in the context of an elastic bar sliding along a deformable planar beam.

It is useful to collect all the virtual displacements of element $A$ into a vector of virtual slave displacements $\delta \mathbf{p}^{A}$. Likewise, stacking the virtual released displacement $\delta \mathbf{Y}$ on top of the vector of virtual master displacements (of both elements) gives the vector of virtual released and master displacements $\delta \mathbf{p}_{R m}^{A}$. Note that in the present terminology, for all the nodes of element $A$ apart from the last one, the master and the slave virtual displacements are identical. These vectors are given explicitly as

$$
\delta \mathbf{p}^{A}=\left\{\begin{array}{c}
\delta \mathbf{p}_{1}^{A} \\
\vdots \\
\delta \mathbf{p}_{N_{A}}^{A}
\end{array}\right\} \quad \text { and } \quad \delta \mathbf{p}_{R m}^{A}=\left\{\begin{array}{c}
\delta \mathbf{Y} \\
\delta \mathbf{p}_{1}^{A} \\
\vdots \\
\delta \mathbf{p}_{N_{A}}^{A} \\
\delta \mathbf{p}_{1}^{B} \\
\vdots \\
\delta \mathbf{p}_{N_{B}}^{B}
\end{array}\right\}
$$

The two vectors can now be related by means of Eq. (13) as

$$
\delta \mathbf{p}^{A}=\mathbf{N} \delta \mathbf{p}_{R m}^{A},
$$


with

$$
\mathbf{N}=\left[\begin{array}{cccccccc}
\mathbf{0}_{3 \times 2} & \mathbf{I} & \ldots & \mathbf{0} & \mathbf{0} & \mathbf{0} & \ldots & \mathbf{0} \\
\vdots & \vdots & \ddots & \vdots & \vdots & \vdots & \ddots & \vdots \\
\mathbf{0}_{3 \times 2} & \mathbf{0} & \ldots & \mathbf{I} & \mathbf{0} & \mathbf{0} & \ldots & \mathbf{0} \\
\mathbf{F}_{B_{1}} & \mathbf{0} & \ldots & \mathbf{0} & \mathbf{0} & I_{B_{1}}^{1} \mathbf{I} & \ldots & I_{B_{1}}^{N_{B}} \mathbf{I}
\end{array}\right]
$$

Eq. (14) provides the essence of the master slave relationship.

\subsection{Finite element assembly: definition of the coupling element}

Let us recast the discretised weak form in (12) as

$$
G^{h}(\mathbf{x}, \delta \mathbf{p})=\delta \mathbf{p}^{A} \cdot \mathbf{g}^{A}+\delta \mathbf{p}^{B} \cdot \mathbf{g}^{B}=0,
$$

where $\delta \mathbf{p}^{I}=\left\{\delta \mathbf{p}_{1}^{I} \ldots \delta \mathbf{p}_{N_{I}}^{I}\right\}$ and $\mathbf{g}^{I}=\left\{\mathbf{g}^{I, 1} \ldots \mathbf{g}^{I, N_{I}}\right\}$ are the elemental virtual displacement vector and the elemental residual vector of element $I, I=A, B$.

It is at this stage that the derived relationship (14) should be utilised. Substituting it into (16) gives

$$
G^{h}(\mathbf{x}, \delta \mathbf{p})=\delta \mathbf{p}_{R m}^{A} \cdot \mathbf{N}^{\mathrm{T}} \mathbf{g}^{A}+\delta \mathbf{p}^{B} \cdot \mathbf{g}^{B}=0,
$$

and we see that the virtual work performed by element $A$ can be expressed as the dot product of a new set of master and released degrees of freedom, $\delta \mathbf{p}_{R m}^{A}$, and an extended residual work-conjugate to them, which is given by

$$
\mathbf{g}_{R m}^{A}=\mathbf{N}^{\mathrm{T}} \mathbf{g}^{A}=\left\{\begin{array}{c}
\mathbf{F}_{B_{1}}^{\mathrm{T}} \mathbf{g}^{A, N_{A}} \\
\mathbf{g}^{A, 1} \\
\vdots \\
\mathbf{g}^{A, N_{A}-1} \\
\mathbf{0} \\
\mathbf{L}^{\mathrm{T}} \mathbf{g}^{A, N_{A}}
\end{array}\right\},
$$

with

$$
\mathbf{L}=\left[\begin{array}{lll}
I_{B_{1}}^{1} \mathbf{I} & \ldots & I_{B_{1}}^{N_{B}} \mathbf{I}
\end{array}\right]
$$

Let us define a coupling element as the element whose elemental displacement vector is $\delta \mathbf{p}_{R m}$. Such a coupling element has the following degrees of freedom: displacements of the nodes in element $A$, the released displacements of node $N_{A}$ and the degrees of freedom of all the nodes in element $B$. The extended residual $\mathbf{g}_{R m}^{A}$ of the coupling element is assembled into the global residual in accordance with the pattern given by $\delta \mathbf{p}_{R m}^{A}$. We emphasise that $\mathbf{g}_{R m}^{A}$ depends on the residual $\mathbf{g}^{A}$ of element $A$ and the terms $\mathbf{F}_{B_{1}}$ and $\mathbf{L}$ which depend on element $B$. Eq. (17) can now be solved using the Newton Raphson iterative procedure in a standard manner.

It is now obvious that if the sliding is not limited to the surface of a single element, we only need to evaluate $\mathbf{F}$ and $\mathbf{L}$ at the contact point of a newly contacted element in order to formulate the new coupling element.

The concepts presented here will now be applied to the mechanics of 3D beams, where additional complexities exist due to the presence of large 3D rotations. The issue of the dynamically changing coupling element will also be dealt with in more detail in the context of sliding between 3D beams. 


\section{Sliding joints in 3D beams}

In this section we apply the concepts from Section 2 to the geometrically exact Reissner Simo beams (Reissner, 1972; Simo, 1985). We will first describe the kinematics of two beams in contact, and then derive the equilibrium equations under the contact condition. The discretised weak form will now include a set of virtual displacements and virtual rotations. Due to the specific kinematics of the beams, a new contact condition relating the torques at the contact point will have to be defined.

\subsection{Beam kinematics}

Let us parametrise the position of any material point $P$ of a beam (a body with one dimension much larger than the other two) by three parameters $\left\{X^{1}, X^{2}, X^{3}\right\}$, where $X^{1}=X$ is the arc-length coordinate along the centroid axis of the beam, and $X^{2}$ and $X^{3}$ are the coordinates of the point on the cross-section $\mathscr{A}(X)$ at $X^{1}$. We will represent the current position at time $t$ of the point $P\left(X^{1}, X^{2}, X^{3}\right)$ in the ambient space by a mapping $\phi_{t}\left(X^{i}\right)=\mathbf{x}\left(X^{i}, t\right)$, where $\phi_{t}$ is defined as

$$
\phi_{t}:[0, L] \times \mathscr{A}(s) \times \mathbb{R}_{+} \rightarrow \mathbb{R}^{3} .
$$

Let us introduce an inertial frame $\mathbf{e}_{i}$ and a moving frame $\mathbf{g}_{i}(X, t), i=1,2,3$, rigidly attached to the crosssection of the beam. We also assume that the undeformed configuration corresponds to a straight beam, and define the (undeformed) reference configuration as the mapping $\chi:[0, L] \times \mathscr{A}(X) \rightarrow \mathbb{R}^{3}$ such that the point $P$ is represented by $\mathbf{X}\left(X^{i}\right)=\chi(P)=X^{i} \mathbf{e}_{i}$ (see Fig. 6).

By assuming the undeformability of the cross-section (beam hypothesis) and according to the Reissner Simo beam theory, the kinematics of the beam at time $t$ will be completely defined by the position of the points of the centroid axis $\mathbf{r}(X, t)$, and the orientations of the cross-section. The latter is represented by a proper orthogonal transformation $\boldsymbol{\Lambda}(X, t) \in S O(3)\left(\operatorname{det} \boldsymbol{\Lambda}=+1\right.$ and $\left.\boldsymbol{\Lambda}^{-1}=\boldsymbol{\Lambda}^{\mathrm{T}}\right)$ such that

$$
\mathbf{g}_{i}(X, t)=\mathbf{\Lambda}(X, t) \mathbf{e}_{i}
$$

The vector $\mathbf{r}$ and the rotation matrix $\boldsymbol{\Lambda}$ allow to express the position of any point $P\left(X^{i}\right)$ of the beam in the (deformed) current configuration as

$$
\mathbf{x}=\mathbf{r}(X, t)+\mathbf{\Lambda}(X, t) \mathbf{Z}\left(X^{2}, X^{3}\right),
$$

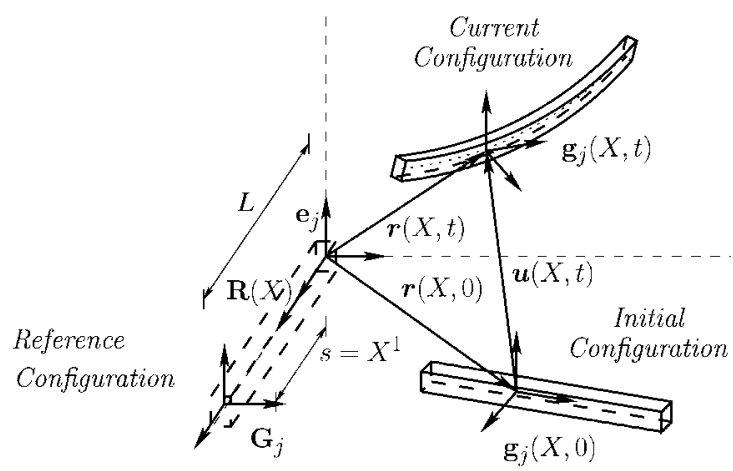

Fig. 6. Beam kinematics. 
where $\mathbf{Z}=X^{2} \mathbf{e}_{2}+X^{3} \mathbf{e}_{3}$ is a vector within the cross-section in the reference configuration. We note that unless stated explicitly, we will denote with capital letters variables referred to the reference configuration (or material quantities), whereas we will use lower-case letters to denote variables in the current configuration (or spatial quantities). We also note that any rotation matrix $\boldsymbol{\Lambda}$ may be expressed as $\boldsymbol{\Lambda}=\exp (\widehat{\boldsymbol{\theta}})$ where $\|\boldsymbol{\theta}\|$ is the rotated angle, $\boldsymbol{\theta} /\|\boldsymbol{\theta}\|$ is the direction around which the rotation takes place (Argyris, 1982), and ( $\widehat{\bullet}$ ) denotes a skew symmetric matrix such that $\widehat{\mathbf{v}} \mathbf{w}=\mathbf{v} \times \mathbf{w}, \forall \mathbf{w}, \mathbf{v} \in \mathbb{R}^{3}$.

The beam kinematics is defined in $\mathbb{R}^{3} \times S O(3)$ and thus the virtual degrees of freedom $\delta \mathbf{p}$ consist of the virtual displacements and rotations which are now the kinematically admissible variations of the configuration space:

$$
\delta \mathbf{p}=\left\{\begin{array}{c}
\delta \mathbf{u} \\
\delta \vartheta
\end{array}\right\}
$$

where $\mathbf{u}(X, t)=\mathbf{r}(X, t)-\mathbf{r}(X, 0)$, and therefore $\delta \mathbf{u}=\delta \mathbf{r}$. The vector $\delta \boldsymbol{\vartheta}$ represents a virtual rotation variation which stems from the variation of matrix $\boldsymbol{\Lambda}$ (Simo, 1985),

$$
\delta \boldsymbol{\Lambda}=\left.\frac{\mathrm{d}}{\mathrm{d} \epsilon}\right|_{\epsilon} \exp (\epsilon \widehat{\delta \boldsymbol{\vartheta}}) \boldsymbol{\Lambda}=\widehat{\delta \boldsymbol{\vartheta}} \boldsymbol{\Lambda}
$$

In order to model the sliding contact between beams, let us consider two beams denoted as $\mathscr{B}^{A}$ and $\mathscr{B}^{B}$ which at time $t$ are in contact at the points of the centroid axis $A_{1}$ and $B_{1}$ respectively as shown in Fig. 7. In contrast to the approach followed in Section 2, the contact condition will now relate the displacements and the rotations of $\mathscr{B}^{A}$ and $\mathscr{B}^{B}$, i.e.

$$
\begin{aligned}
& \mathbf{r}_{t}\left(X_{A_{1}}\right)=\mathbf{r}_{t}\left(X_{B_{1}}\right), \\
& \boldsymbol{\Lambda}_{t}\left(X_{A_{1}}\right)=\boldsymbol{\Lambda}_{t}\left(X_{B_{1}}\right) \boldsymbol{\Lambda}_{\mathrm{rel}},
\end{aligned}
$$

where the matrix $\boldsymbol{\Lambda}_{\text {rel }}$ in (21) relates the orientation of the two beams at the initial configuration: $\boldsymbol{\Lambda}_{\text {rel }}=\boldsymbol{\Lambda}_{0}\left(X_{B_{1}}\right)^{\mathrm{T}} \boldsymbol{\Lambda}_{0}\left(X_{A_{1}}\right)$. Fig. 7 illustrates this situation.

In a similar manner to that given in the previous section, we introduce the following equivalent set of hypotheses consistent with the considered beam model:

h1 At time $t$, beam $\mathscr{B}^{A}$ exerts a force $\mathbf{S}_{B_{1}}$ on point $B^{1}$ of beam $\mathscr{B}^{B}$. This force is taken to be equal in magnitude and opposite in direction to force $\mathbf{S}_{A_{1}}$ exerted by beam $\mathscr{B}^{B}$ on point $A_{1}$ of beam $\mathscr{B}^{A}$ :

$$
\mathbf{S}_{B_{1}}=-\mathbf{S}_{A_{1}} \text {. }
$$

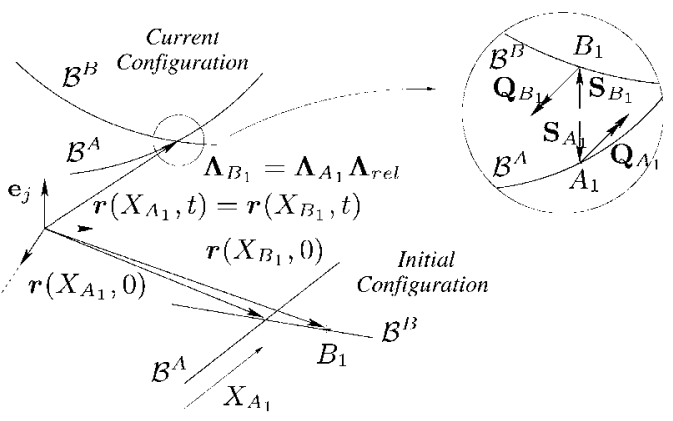

Fig. 7. Kinematics of beams $\mathscr{B}^{A}$ and $\mathscr{B}^{B}$ in contact. 
The beam kinematics, and in particular Eq. (21), make it reasonable to supplement this assumption with the following additional kinetic hypothesis related to the transmission of contact torques.

h2 At time $t$, beam $\mathscr{B}^{A}$ exerts a torque $\mathbf{Q}_{B_{1}}$ on point $B^{1}$ of beam $\mathscr{B}^{B}$. This torque is taken to be equal in magnitude and opposite in direction to torque $\mathbf{Q}_{A_{1}}$ exerted by beam $\mathscr{B}^{B}$ on point $A_{1}$ of beam $\mathscr{B}^{A}$ :

$$
\mathbf{Q}_{B_{1}}=-\mathbf{Q}_{A_{1}} \text {. }
$$

h3 Frictionless bilateral contact is assumed, i.e. the interaction forces between the bodies along the tangent to the centroid lines of beams $\mathscr{B}^{A}$ and $\mathscr{B}^{B}$ will be considered equal to zero:

$$
\mathbf{S}_{A_{1}} \cdot \mathbf{r}_{A_{1}}^{\prime}=0 \text { and } \mathbf{S}_{B_{1}} \cdot \mathbf{r}_{B_{1}}^{\prime}=0 \text {, }
$$

where here and in the rest of the paper, we denote by $(\bullet)^{\prime}$ the derivative of $(\bullet)$ with respect to the parameter $X$.

\subsection{Beam equilibrium equations}

We will consider the governing equations for each one of the beams $\mathscr{B}^{A}$ and $\mathscr{B}^{B}$ separately and deduce the corresponding weak form of the complete system by introducing the infinitesimal form of the sliding condition in a manner analogous to that of Section 2.

Denoting by $A$ and $\rho$ the area of the cross-section and the density of the material, the local equilibrium equations can be written as (Simo, 1985)

$$
\left\{\begin{array}{l}
\dot{\boldsymbol{i}} \\
\dot{\pi}
\end{array}\right\}=\left\{\begin{array}{c}
(\mathbf{\Lambda} \mathbf{N})^{\prime}+\mathbf{s}_{n} \\
(\mathbf{\Lambda} \mathbf{M})^{\prime}+\widehat{\mathbf{r}}^{\prime} \boldsymbol{\Lambda} \mathbf{N}+\mathbf{s}_{m}
\end{array}\right\},
$$

where $\boldsymbol{l}=A \rho \dot{\mathbf{u}}$ and $\boldsymbol{\pi}=\mathbf{\Lambda} \mathbf{J W}$ are the specific translational and angular momenta, and $\mathbf{W}$ and $\mathbf{J}$ are the (material) angular velocity and the tensor of the mass moments of inertia of a cross-section. The vectors $\mathbf{N}=\mathbf{C}_{N} \gamma$ and $\mathbf{M}=\mathbf{C}_{M} \boldsymbol{\kappa}$ are the (material) stress resultants at the cross-section, $\mathbf{C}_{N}$ and $\mathbf{C}_{M}$ are the modulus matrices, $\boldsymbol{\gamma}=\boldsymbol{\Lambda}^{\mathrm{T}} \mathbf{r}^{\prime}-\mathbf{G}_{1}$ and $\widehat{\boldsymbol{\kappa}}=\boldsymbol{\Lambda}^{\mathrm{T}} \boldsymbol{\Lambda}^{\prime}$ are the translational and rotational strain measures, and $\mathbf{s}_{n}$ and $\mathbf{s}_{m}$ are the distributed load and torque per unit of undeformed length applied on $X \in\left[0, L^{I}\right], I=A, B$.

The governing equations must be complemented with the boundary conditions corresponding to the end loads (for simplicity, no prescribed displacements will be considered),

$$
\left\{\begin{array}{c}
\mathbf{\Lambda} \mathbf{N} \\
\mathbf{\Lambda} \mathbf{M}
\end{array}\right\}_{X^{I} 0}=-\left\{\begin{array}{c}
\mathbf{S}_{0}^{I} \\
\mathbf{Q}_{0}^{I}
\end{array}\right\} ; \quad\left\{\begin{array}{c}
\mathbf{\Lambda} \mathbf{N} \\
\mathbf{\Lambda} \mathbf{M}
\end{array}\right\}_{X^{I} L^{I}}=\left\{\begin{array}{l}
\mathbf{S}_{L}^{I} \\
\mathbf{Q}_{L}^{I}
\end{array}\right\} \quad I=A, B
$$

where $\mathbf{S}_{0}^{I}, \mathbf{Q}_{0}^{I}, \mathbf{S}_{L}^{I}$ and $\mathbf{Q}_{L}^{I}$ are the concentrated loads and torques at the two ends of each beam $\mathscr{B}^{I}$. Also, there exist the concentrated force and torque due to the bilateral contact

$$
\left\{\begin{array}{l}
\mathbf{S}_{I_{1}} \\
\mathbf{Q}_{I_{1}}
\end{array}\right\}=\lim _{\epsilon \rightarrow 0} \int_{X_{I_{1}}-\epsilon}^{X_{I_{1}}+\epsilon}\left\{\begin{array}{l}
\mathbf{s}_{n} \\
\mathbf{s}_{m}
\end{array}\right\} \mathrm{d} X \quad I=A, B
$$

The weak form of the equilibrium equations is obtained by multiplying (22) with the virtual displacements (or test functions) $\delta \mathbf{p}$ and integrating over the length $L^{I}$ of each beam $I=A, B$,

$$
\begin{aligned}
G(\mathbf{r}, \boldsymbol{\Lambda}, \delta \mathbf{u}, \delta \boldsymbol{\vartheta}) \equiv & \sum_{I} \int_{A, B} \delta \mathbf{p} \cdot\left\{\begin{array}{c}
\dot{\boldsymbol{i}}-(\mathbf{\Lambda} \mathbf{N})^{\prime} \\
\dot{\pi}-\widehat{\mathbf{r}}^{\prime} \boldsymbol{\Lambda} \mathbf{N}-(\mathbf{\Lambda} \mathbf{M})^{\prime}
\end{array}\right\} \mathrm{d} X-\sum_{I A, B}\left[\int_{L^{I} \backslash X_{I_{1}}} \delta \mathbf{p} \cdot\left\{\begin{array}{l}
\mathbf{s}_{n} \\
\mathbf{s}_{m}
\end{array}\right\} \mathrm{d} X\right. \\
& \left.+\lim _{\epsilon \rightarrow 0} \int_{X_{I_{1}}-\epsilon}^{X_{I_{1}}+\epsilon} \delta \mathbf{p} \cdot\left\{\begin{array}{l}
\mathbf{s}_{n} \\
\mathbf{s}_{m}
\end{array}\right\}_{X_{I_{1}}} \mathrm{~d} X\right] \\
= & 0 .
\end{aligned}
$$


In order to simplify this expression, we note first that the last term corresponds to the virtual work done by the external load $\left\{\mathbf{S}_{I_{1}} \mathbf{Q}_{I_{1}}\right\}$ :

$$
\lim _{\epsilon \rightarrow 0} \int_{X_{I_{1}}-\epsilon}^{X_{I_{1}}+\epsilon} \delta \mathbf{p} \cdot\left\{\begin{array}{l}
\mathbf{s}_{n} \\
\mathbf{s}_{m}
\end{array}\right\}_{X_{I_{1}}} \mathrm{~d} X=\delta \mathbf{p}_{I_{1}} \cdot\left\{\begin{array}{l}
\mathbf{S}_{I_{1}} \\
\mathbf{Q}_{I_{1}}
\end{array}\right\} \quad I=A, B .
$$

By using this result, integrating by parts the terms with $(\boldsymbol{\Lambda N})^{\prime}$ and $(\boldsymbol{\Lambda} \mathbf{M})^{\prime}$ and substituting the boundary conditions (23) and (24), the weak form can be expressed as:

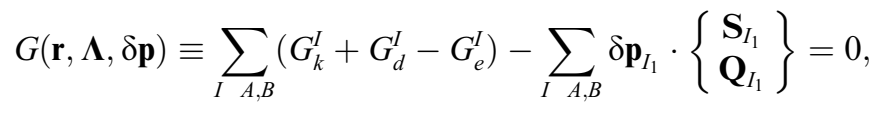

where $\delta \mathbf{p}_{I_{1}}$ are the virtual displacements and rotations evaluated at points $I_{1}$, and $G_{k}^{I}, G_{d}^{I}$ and $G_{e}^{I}$ are the internal, dynamic and external contributions to the weak form, which are given by

$$
\begin{aligned}
& G_{k}^{I}=\int_{L^{I}} \delta \mathbf{p}^{\prime} \cdot\left\{\begin{array}{c}
\boldsymbol{\Lambda} \mathbf{N} \\
\boldsymbol{\Lambda} \mathbf{M}
\end{array}\right\} \mathrm{d} X-\int_{L^{I}} \delta \mathbf{p} \cdot\left\{\begin{array}{c}
\mathbf{0} \\
\widehat{\mathbf{r}}^{\prime} \boldsymbol{\Lambda} \mathbf{N}
\end{array}\right\} \mathrm{d} X, \\
& G_{d}^{I}=\int_{L^{I}} \delta \mathbf{p} \cdot\left\{\begin{array}{l}
\boldsymbol{i} \\
\dot{\pi}
\end{array}\right\} \mathrm{d} X, \\
& G_{e}^{I}=\int_{L^{I}} \delta \mathbf{p} \cdot\left\{\begin{array}{l}
\mathbf{s}_{n} \\
\mathbf{s}_{m}
\end{array}\right\} \mathrm{d} X+\delta \mathbf{p}_{L}^{I} \cdot\left\{\begin{array}{c}
\mathbf{S}_{L}^{I} \\
\mathbf{Q}_{L}^{I}
\end{array}\right\}+\delta \mathbf{p}_{0}^{I} \cdot\left\{\begin{array}{l}
\mathbf{S}_{0}^{I} \\
\mathbf{Q}_{0}^{I}
\end{array}\right\} .
\end{aligned}
$$

\subsection{Infinitesimal kinematic contact conditions}

Let the deformed configuration $\phi_{t}$ be perturbed by a kinematically admissible virtual displacement and rotation $\epsilon \delta$. We will assume that contact point $A_{1}$ remains in contact with beam $\mathscr{B}^{B}$ permanently, whereas the contact point of beam $\mathscr{B}^{B}$ changes during the perturbation (see Fig. 8): in the deformed configuration, the contact is established at point $B_{1}$, while in the perturbed configuration it is established at $B_{2}$.

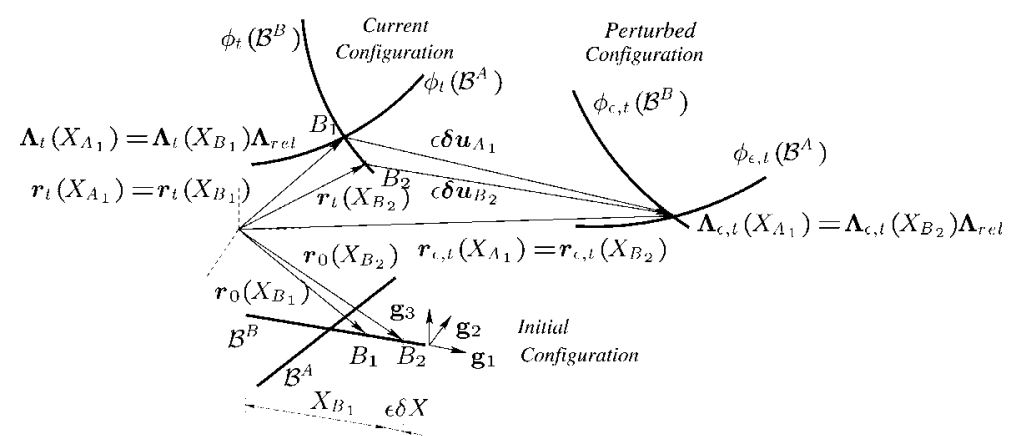

Fig. 8. Initial, current and perturbed configuration of beams $\mathscr{B}^{A}$ and $\mathscr{B}^{B}$. 
The contact conditions (20) and (21) in the perturbed configuration are given by

$$
\begin{aligned}
& \mathbf{r}_{\epsilon, t}\left(X_{A_{1}}\right)=\mathbf{r}_{\epsilon, t}\left(X_{B_{2}}\right), \\
& \boldsymbol{\Lambda}_{\epsilon, t}\left(X_{A_{1}}\right)=\boldsymbol{\Lambda}_{\epsilon, t}\left(X_{B_{2}}\right) \boldsymbol{\Lambda}_{\text {rel }} .
\end{aligned}
$$

which provide the following relationships between virtual quantities:

$$
\begin{aligned}
& \left.\delta \mathbf{u}_{A_{1}} \equiv \frac{\mathrm{d}}{\mathrm{d} \epsilon}\right|_{\epsilon} \mathbf{r}_{\epsilon, t}\left(X_{A_{1}}\right)=\left.\frac{\mathrm{d}}{\mathrm{d} \epsilon}\right|_{\epsilon}\left[\mathbf{r}_{t}\left(X_{B_{1}}+\epsilon \delta X\right)+\epsilon \delta \mathbf{u}\left(X_{B_{1}}+\epsilon \delta X\right)\right]=\mathbf{r}_{t}^{\prime}\left(X_{B_{1}}\right) \delta X+\delta \mathbf{u}_{B_{1}}, \\
& \left.\widehat{\delta \boldsymbol{\vartheta}}_{A_{1}} \boldsymbol{\Lambda}_{t}\left(X_{A_{1}}\right) \equiv \frac{\mathrm{d}}{\mathrm{d} \epsilon}\right|_{\epsilon} \boldsymbol{\Lambda}_{\epsilon, t}\left(X_{A_{1}}\right)=\left.\frac{\mathrm{d}}{\mathrm{d} \epsilon}\right|_{\epsilon}\left[\exp \left(\epsilon \widehat{\delta \boldsymbol{\vartheta}}\left(X_{B_{1}}+\epsilon \delta X_{B_{1}}\right)\right) \boldsymbol{\Lambda}_{t}\left(X_{B_{1}}+\epsilon \delta X\right) \boldsymbol{\Lambda}_{\text {rel }}\right] \\
& =\left(\widehat{\mathbf{k}}_{B_{1}} \delta X+\widehat{\delta \boldsymbol{\vartheta}}_{B_{1}}\right) \boldsymbol{\Lambda}_{t}\left(X_{B_{1}}\right) \boldsymbol{\Lambda}_{\text {rel }},
\end{aligned}
$$

where $\delta X$ is the variation of the contact point on the reference configuration and $\mathbf{k}_{B_{1}}=\boldsymbol{\Lambda}\left(X_{B_{1}}\right) \boldsymbol{\kappa}_{B_{1}}$ is the (spatial) curvature of the beam evaluated at $X_{B_{1}}$. Using this result along with the contact condition (21) gives the important relationships

$$
\begin{aligned}
& \delta \mathbf{u}_{A_{1}}=\mathbf{r}_{B_{1}}^{\prime} \delta X+\delta \mathbf{u}_{B_{1}}, \\
& \delta \boldsymbol{\vartheta}_{A_{1}}=\mathbf{k}_{B_{1}} \delta X+\delta \boldsymbol{\vartheta}_{B_{1}},
\end{aligned}
$$

where $\mathbf{r}_{B_{1}}^{\prime}=\mathbf{r}_{t}^{\prime}\left(X_{B_{1}}\right)$. Substituting (28) into (25), and making use of hypotheses h1 h3, provides the following result:

$$
G(\mathbf{r}, \Lambda, \delta \mathbf{p})=\sum_{I A, B}\left(G_{k}^{I}+G_{d}^{I}-G_{e}^{I}\right)-\mathbf{k}_{B_{1}} \cdot \mathbf{Q}_{A_{1}} \delta X=0
$$

in which the interaction torque between the two beams, $\mathbf{Q}_{A_{1}}$, will be related to the vector of nodal residuals upon the introduction of the spatial discretisation in Section 3.4.

We will extend Eq. $(28)_{2}$ to contact conditions with a variable relative rotation between the two beams. This will allow us to model joints with released rotations like a cylindrical joint sliding along a flexible beam. The rotational contact condition set in (21) assumes that the relative rotation of beams $\mathscr{B}^{A}$ and $\mathscr{B}^{B}$ is maintained constant throughout the motion. In case when this relative rotation is not constant, a new rotation matrix $\boldsymbol{\Lambda}_{R}$ is introduced which then redefines the rotation at point $A_{1}$ of beam $\mathscr{B}^{A}$ to be

$$
\boldsymbol{\Lambda}\left(X_{A_{1}}\right)=\boldsymbol{\Lambda}\left(X_{B_{1}}\right) \boldsymbol{\Lambda}_{R} \boldsymbol{\Lambda}_{\text {rel }} .
$$

The matrix $\boldsymbol{\Lambda}_{R}$ measures the released rotation at $X_{A_{1}}$ with respect to the rotation $\boldsymbol{\Lambda}\left(X_{B_{1}}\right)$ (rotation at point $B_{1}$ of beam $\mathscr{B}^{B}$ ), without accounting of the initial relative rotation $\boldsymbol{\Lambda}_{\text {rel }}$. We note that we use the released rotation matrix $\boldsymbol{\Lambda}_{R}$ measured in the moving frame $\mathbf{g}_{i}$ of beam $\mathscr{B}^{B}$. Eq. (30) is linearised in the standard way to give

$$
\delta \boldsymbol{\vartheta}_{A 1}=\mathbf{k}_{B_{1}} \delta X+\delta \boldsymbol{\vartheta}_{B_{1}}+\boldsymbol{\Lambda}_{B_{1}} \delta \boldsymbol{\vartheta}_{R}
$$

with $\delta \boldsymbol{\vartheta}_{R}$ as the virtual released rotation and $\boldsymbol{\Lambda}_{B_{1}}=\boldsymbol{\Lambda}\left(X_{B_{1}}\right)$. Instead of using the vector $\delta \boldsymbol{\vartheta}_{R}$ we will rather use the variation $\delta \boldsymbol{\theta}_{R}$ of the rotation vector $\boldsymbol{\theta}_{R}$ in $\boldsymbol{\Lambda}_{R}=\exp \widehat{\boldsymbol{\theta}_{R}}$. This choice is necessitated by the nature of some kinds of joints, the kinematics of which may not be defined using $\delta \boldsymbol{\vartheta}_{R}$ (Jelenić and Crisfield, 2001). The relation between $\delta \vartheta$ and $\delta \boldsymbol{\theta}$ can be deduced by considering the following equation

$$
\left.\frac{\mathrm{d}}{\mathrm{d} \epsilon}\right|_{\epsilon 0} \exp (\epsilon \widehat{\delta \boldsymbol{\vartheta}})=\left.\frac{\mathrm{d}}{\mathrm{d} \epsilon}\right|_{\epsilon} \exp (\boldsymbol{\theta}+\epsilon \widehat{\delta \boldsymbol{\theta}})
$$

which gives rise to (see e.g. Cardona and Géradin, 1988; Ritto-Corrêa and Camotim, 2002; Simo and Vu-Quoc, 1986) 


$$
\delta \boldsymbol{\vartheta}=\mathbf{T}^{-1}(\boldsymbol{\theta}) \delta \boldsymbol{\theta} ; \quad \mathbf{T}^{-1}(\boldsymbol{\theta})=\mathbf{I}+\frac{1-\cos \|\boldsymbol{\theta}\|}{\|\boldsymbol{\theta}\|^{2}} \widehat{\boldsymbol{\theta}}+\left(1-\frac{\sin \|\boldsymbol{\theta}\|}{\|\boldsymbol{\theta}\|}\right) \frac{\widehat{\boldsymbol{\theta}}^{2}}{\|\boldsymbol{\theta}\|^{2}} .
$$

Substituting $\delta \boldsymbol{\vartheta}_{R}$ into (31), we obtain

$$
\delta \boldsymbol{\vartheta}_{A_{1}}=\mathbf{k}_{B_{1}} \delta X+\boldsymbol{\Lambda}_{B_{1}} \mathbf{T}_{R}^{-1} \delta \boldsymbol{\theta}_{R}+\delta \boldsymbol{\vartheta}_{B_{1}},
$$

where $\mathbf{T}_{R}^{-1}=\mathbf{T}^{-1}\left(\boldsymbol{\theta}_{R}\right)$. By using relation (32) instead of (28), a new term arises in the weak form (29), which is given by

$$
G(\mathbf{r}, \boldsymbol{\Lambda}, \delta \mathbf{p}) \equiv \sum_{I}\left(G_{k}^{I}+G_{d}^{I}-G_{e}^{I}\right)-\mathbf{k}_{B_{1}} \cdot \mathbf{Q}_{A_{1}} \delta X+\boldsymbol{\Lambda}_{B_{1}} \mathbf{T}_{R}^{-1} \delta \boldsymbol{\theta}_{R} \cdot \mathbf{Q}_{B_{1}}=0
$$

where the last term represents the virtual work done by the contact torque $\mathbf{Q}_{B_{1}}$ under a virtual released rotation $\delta \boldsymbol{\vartheta}_{r}=\boldsymbol{\Lambda}_{B_{1}} \delta \boldsymbol{\vartheta}_{R}$. If no friction is considered at the contact point under a relative rotation, no reaction torque exist in the direction of the virtual released rotation and therefore this terms vanishes, i.e. $\delta \boldsymbol{\vartheta}_{r} \cdot \mathbf{Q}_{B_{1}}=0$. We are in fact assuming an equivalent hypothesis to h3 for the case of rotations.

\subsection{Finite element discretisation}

Let us discretise the beams $\mathscr{B}^{A}$ and $\mathscr{B}^{B}$ using $N_{A}$ and $N_{B}$ nodes respectively. The vector $\delta \mathbf{p}(X)$ will be discretised by using the standard Lagrangian polynomials in (11) which now depend exclusively on the parameter $X$, i.e.

$$
\delta \mathbf{p}^{h}(X)=I^{j}(X) \delta \mathbf{p}_{j} .
$$

By replacing the vector $\delta \mathbf{p}$ in (29) with $\delta \mathbf{p}^{h}$, the discretised weak form $G^{h}$ is readily obtained as

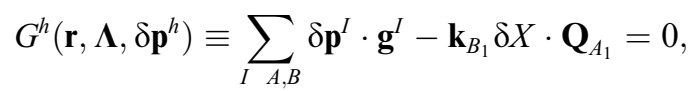

where $\delta \mathbf{p}^{I}=\left\{\delta \mathbf{p}_{1}^{I} \ldots \delta \mathbf{p}_{N_{I}}^{I}\right\}$ is the vector of elemental virtual displacements and rotations and $\mathbf{g}^{I}=\left\{\mathbf{g}^{I, 1} \ldots \mathbf{g}^{I, N_{I}}\right\}$ is the elemental residual vector of element $I$. Each nodal component of $\mathbf{g}^{I}$ is the contribution of the internal, dynamic and external nodal residual vectors $\mathbf{q}_{k}^{I, j}, \mathbf{q}_{d}^{I, j}$ and $\mathbf{q}_{e}^{I, j}$ respectively, i.e. $\mathbf{g}^{I, j}=\mathbf{q}_{k}^{I, j}+\mathbf{q}_{d}^{I, j}-\mathbf{q}_{e}^{I, j}$. They are obtained from (26) as

$$
\begin{aligned}
\mathbf{q}_{k}^{I, j} & =\int_{L^{I}} I^{j j}\left\{\begin{array}{c}
\boldsymbol{\Lambda} \mathbf{N} \\
\boldsymbol{\Lambda} \mathbf{M}
\end{array}\right\} \mathrm{d} X-\int_{L^{I}} I^{j}\left\{\begin{array}{c}
\mathbf{0} \\
\widehat{\mathbf{r}}^{\prime} \boldsymbol{\Lambda} \mathbf{N}
\end{array}\right\} \mathrm{d} X, \\
\mathbf{q}_{d}^{I, j} & =\int_{L^{I}} I^{j}\left\{\begin{array}{l}
\dot{\boldsymbol{l}} \\
\dot{\pi}
\end{array}\right\} \mathrm{d} X, \\
\mathbf{q}_{e}^{I, j} & =\int_{L^{I}} I^{j}\left\{\begin{array}{l}
\mathbf{s}_{n} \\
\mathbf{s}_{m}
\end{array}\right\} \mathrm{d} X+\delta_{1}^{j}\left\{\begin{array}{l}
\mathbf{S}_{0}^{I} \\
\mathbf{Q}_{0}^{I}
\end{array}\right\}+\delta_{N_{I}}^{j}\left\{\begin{array}{l}
\mathbf{S}_{L}^{I} \\
\mathbf{Q}_{L}^{I}
\end{array}\right\} .
\end{aligned}
$$

The master slave relationship (28) $)_{1}$ and (32) may be rewritten as

$$
\delta \mathbf{p}_{N_{A}}=\left\{\begin{array}{c}
\mathbf{r}_{B_{1}}^{\prime} \\
\mathbf{k}_{B_{1}}
\end{array}\right\}\left(\delta \mathbf{u}_{R} \cdot \mathbf{G}_{1}\right)+\left\{\begin{array}{c}
\mathbf{0} \\
\boldsymbol{\Lambda}_{B_{1}} \mathbf{T}_{R}^{-1} \delta \boldsymbol{\theta}_{R}
\end{array}\right\}+I_{B}^{j} \delta \mathbf{p}_{j},
$$

where $\delta \mathbf{u}_{R}=\left\{\begin{array}{lll}\delta X & 0 & 0\end{array}\right\}$ is the vector of released displacements, and as in the general case of elastodynamics, we assume that the sliding point on element $A$ corresponds to node $N_{A}$ throughout the motion. As in Section 2.3, we will define the slave and master and released vectors of virtual displacements and rotations as 


$$
\delta \mathbf{p}^{A}=\left\{\begin{array}{c}
\delta \mathbf{p}_{1}^{A} \\
\vdots \\
\delta \mathbf{p}_{N_{A}}^{A}
\end{array}\right\} \quad \text { and } \quad \delta \mathbf{p}_{R m}^{A}=\left\{\begin{array}{c}
\delta \mathbf{p}_{R} \\
\delta \mathbf{p}_{1}^{A} \\
\vdots \\
\delta \mathbf{p}_{N_{A}}^{A} \\
\delta \mathbf{p}_{1}^{B} \\
\vdots \\
\delta \mathbf{p}_{N_{B}}^{B}
\end{array}\right\}
$$

where $\delta \mathbf{p}_{R}$ is the vector composed of the virtual released displacement and rotation $\delta \mathbf{u}_{R}$ and $\delta \boldsymbol{\theta}_{R}$. Using (36) we can relate the two vectors as

$$
\delta \mathbf{p}^{A}=\mathbf{N}^{*} \delta \mathbf{p}_{R m}^{A},
$$

with

$$
\mathbf{N}^{*}=\left[\begin{array}{cccccccc}
\mathbf{0} & \mathbf{I} & \ldots & \mathbf{0} & \mathbf{0} & \mathbf{0} & \ldots & \mathbf{0} \\
\vdots & \vdots & \ddots & \vdots & \vdots & \vdots & \ddots & \vdots \\
\mathbf{0} & \mathbf{0} & \ldots & \mathbf{I} & \mathbf{0} & \mathbf{0} & \ldots & \mathbf{0} \\
\mathbf{R}_{B}^{*} & \mathbf{0} & \ldots & \mathbf{0} & \mathbf{0} & I_{B}^{1} \mathbf{I} & \ldots & I_{B}^{N_{B}} \mathbf{I}
\end{array}\right] ; \quad \mathbf{R}_{B}^{*}=\left[\begin{array}{cc}
\mathbf{r}_{B_{1}}^{\prime} \otimes \mathbf{G}_{1} & \mathbf{0} \\
\mathbf{k}_{B_{1}} \otimes \mathbf{G}_{1} & \boldsymbol{\Lambda}_{B} \mathbf{T}_{R}^{-1}
\end{array}\right]
$$

and where the matrices $\mathbf{0}$ and $\mathbf{I}$ are the $6 \times 6$ zero and unit matrices.

In order to deal with the term with the contact torque in the weak form $G^{h}$, we insert the master slave relationship (37) into Eq. (34), which leads to

$$
G^{h}(\mathbf{r}, \boldsymbol{\Lambda}, \delta \mathbf{p})=\delta \mathbf{p}_{R m}^{A} \cdot \mathbf{N}^{* \mathrm{~T}} \mathbf{g}^{A}+\delta \mathbf{p}^{B} \cdot \mathbf{g}^{B}-\delta \mathbf{u}_{R} \cdot\left(\mathbf{G}_{1} \otimes \mathbf{k}_{B_{1}}\right) \mathbf{Q}_{A_{1}} \cdot
$$

We now note that the discretised weak form $G^{h}$ in (34) can be split in two weak forms $G_{A}^{h}$ and $G_{B}^{h}$ given by

$$
\begin{aligned}
& G_{A}^{h}(\mathbf{r}, \boldsymbol{\Lambda}, \delta \mathbf{p}) \equiv \delta \mathbf{p}^{A} \cdot \mathbf{g}^{A}-\delta \mathbf{p}_{N_{A}}^{A} \cdot\left\{\begin{array}{l}
\mathbf{S}_{A_{1}} \\
\mathbf{Q}_{A_{1}}
\end{array}\right\}=0, \\
& G_{B}^{h}(\mathbf{r}, \boldsymbol{\Lambda}, \delta \mathbf{p}) \equiv \delta \mathbf{p}^{B} \cdot \mathbf{g}^{B}-I_{B}^{j} \delta \mathbf{p}_{j}^{B} \cdot\left\{\begin{array}{l}
\mathbf{S}_{B_{1}} \\
\mathbf{Q}_{B_{1}}
\end{array}\right\}=0
\end{aligned}
$$

which correspond to the application of the virtual work principle to each beam separately. The weak form in (39) is in fact the sum of the two parts: $G^{h}=G_{A}^{h}+G_{B}^{h}=0$. Gathering the terms multiplying the virtual rotations of node $N_{A}$ in Eq. (40) $)_{1}$ we obtain the following identity

$$
\mathbf{g}_{m}^{N_{A}}-\mathbf{Q}_{A_{1}}=\mathbf{0},
$$

where $\mathbf{g}_{m}^{N_{A}}$ is the rotational part of the residual vector $\mathbf{g}^{N_{A}}$. Substituting this equation into (39) and making use of (38) now gives

$$
G^{h}(\mathbf{r}, \boldsymbol{\Lambda}, \delta \mathbf{p})=\delta \mathbf{p}_{R m}^{A} \cdot \mathbf{N}^{\mathrm{T}} \mathbf{g}^{A}+\delta \mathbf{p}^{B} \cdot \mathbf{g}^{B}=0,
$$

with

$$
\mathbf{N}=\left[\begin{array}{cccccccc}
\mathbf{0} & \mathbf{I} & \ldots & \mathbf{0} & \mathbf{0} & \mathbf{0} & \ldots & \mathbf{0} \\
\vdots & \vdots & \ddots & \vdots & \vdots & \vdots & \ddots & \vdots \\
\mathbf{0} & \mathbf{0} & \ldots & \mathbf{I} & \mathbf{0} & \mathbf{0} & \ldots & \mathbf{0} \\
\mathbf{R}_{B} & \mathbf{0} & \ldots & \mathbf{0} & \mathbf{0} & I_{B}^{1} \mathbf{I} & \ldots & I_{B}^{N_{B}} \mathbf{I}
\end{array}\right] ; \quad \mathbf{R}_{B}=\left[\begin{array}{cc}
\mathbf{r}_{B}^{\prime} \otimes \mathbf{G}_{1} & \mathbf{0} \\
\mathbf{0} & \boldsymbol{\Lambda}_{B} \mathbf{T}_{R}^{-1}
\end{array}\right]
$$


Now we can proceed as in Section 2 by defining the coupling element as an element with $6 \times\left(1+N_{A}+N_{B}\right)$ degrees of freedom and a dynamic residual vector given by the product $\mathbf{N}^{\mathrm{T}} \mathbf{g}^{A}$,

$$
\mathbf{g}_{R m}^{A}=\mathbf{N}^{\mathrm{T}} \mathbf{g}^{A}=\left\{\begin{array}{c}
\mathbf{R}_{B}^{\mathrm{T}} \mathbf{g}^{N_{A}} \\
\mathbf{g}^{A, 1} \\
\vdots \\
\mathbf{g}^{A, N_{A}-1} \\
\mathbf{0} \\
\mathbf{L}^{\mathrm{T}} \mathbf{g}^{N_{A}}
\end{array}\right\}, \quad \text { with } \quad \mathbf{L}=\left[\begin{array}{lll}
I_{B}^{1} \mathbf{I} & \cdots & I_{B}^{N_{B}} \mathbf{I}
\end{array}\right] .
$$

This extended residual $\mathbf{g}_{R m}^{A}$ is analogous to the residual in (18) in the case of elastodynamics and depends obviously on vector $\mathbf{g}^{A}$ and on the kinematics of the contacted element by means of matrix $\mathbf{R}_{B}$ and the values of the interpolating functions $I_{B}^{j}$.

\subsection{Numerical time integration}

In order to process the dynamic residual of the coupling element (43) (and indeed the dynamic residuals of all the other elements in the structure), special attention must be paid to the vector of inertial forces $\mathbf{q}_{d}^{I, j}$ within the dynamic residual. It is obvious from (35) that this vector contains the time derivatives of the specific momenta $\boldsymbol{l}=A \rho \dot{\mathbf{u}}$ and $\boldsymbol{\pi}=\boldsymbol{\Lambda} \mathbf{J W}$, i.e.

$$
\dot{\boldsymbol{l}}=A \rho \ddot{\mathbf{u}} \quad \text { and } \quad \dot{\pi}=\boldsymbol{\Lambda}(\mathbf{W} \times \mathbf{J W}+\mathbf{J A}),
$$

where $\mathbf{A}$ is the vector of (body) angular accelerations of a cross-section. The dynamic residual therefore forms a set of ordinary second-order differential equations, which need to be integrated in time in order to provide a numerical solution. There exist many different schemes for the numerical integration of velocities and accelerations in a mechanical system and, in the recent years, much progress has been made in the design of problem-dependent time-integration techniques which inherit some of the important properties of motion from the underlying continuum case. In a companion paper (Muñoz and Jelenić, in preparation), we will attempt to make a contribution to this topic in relation to the problem of dynamics of flexible beams with sliding joints, but here we limit our attention to a simple and widely used Newmark's two-parameter family of algorithms. In these algorithms, the velocities $\mathbf{v}_{n+1}$ and accelerations $\mathbf{a}_{n+1}$ at time $t_{n+1}$ are defined using the displacements $\mathbf{u}_{n}$, velocities $\mathbf{v}_{n}$ and accelerations $\mathbf{a}_{n}$ at time $t_{n}$, the displacements $\mathbf{u}_{n+1}$ at time $t_{n+1}$ and the parameters $\beta$ and $\gamma$ as (Newmark, 1959)

$$
\begin{aligned}
& \mathbf{v}_{n+1}=\frac{\gamma}{\beta \Delta t}\left(\mathbf{u}_{n+1}-\mathbf{u}_{n}\right)+\left(1-\frac{\gamma}{\beta}\right) \mathbf{v}_{n}+\Delta t\left(1-\frac{\gamma}{2 \beta}\right) \mathbf{a}_{n}, \\
& \mathbf{a}_{n+1}=\frac{1}{\beta \Delta t^{2}}\left[\mathbf{u}_{n+1}-\mathbf{u}_{n}-\Delta t \mathbf{v}_{n}-\Delta t^{2}\left(\frac{1}{2}-\beta\right) \mathbf{a}_{n}\right] .
\end{aligned}
$$

The application of such an integration to angular velocities and accelerations only makes sense if performed in a body-attached frame, i.e. in the material setting (Simo and Wong, 1991). In this way we obtain

$$
\begin{aligned}
& \mathbf{W}_{n+1}=\frac{\gamma}{\beta \Delta t} \boldsymbol{\Theta}+\left(1-\frac{\gamma}{\beta}\right) \mathbf{W}_{n}+\Delta t\left(1-\frac{\gamma}{2 \beta}\right) \mathbf{A}_{n}, \\
& \mathbf{A}_{n+1}=\frac{1}{\beta \Delta t^{2}}\left[\boldsymbol{\Theta}-\Delta t \mathbf{W}_{n}-\Delta t^{2}\left(\frac{1}{2}-\beta\right) \mathbf{A}_{n}\right],
\end{aligned}
$$

where $\mathbf{W}_{n}, \mathbf{A}_{n}, \mathbf{W}_{n+1}$ and $\mathbf{A}_{n+1}$ are the material angular velocities and accelerations at times $t_{n}$ and $t_{n+1}$ and $\boldsymbol{\Theta}$ is the material rotational vector between configurations at times $t_{n}$ and $t_{n+1}$. This material rotational vector is related to the spatial incremental rotational vector through $\boldsymbol{\Theta}=\boldsymbol{\Lambda}_{n}^{\mathrm{T}} \boldsymbol{\theta}=\boldsymbol{\Lambda}_{n+1}^{\mathrm{T}} \boldsymbol{\theta}$. Performing the 
time-integration of velocities and accelerations as given here turns all of the contributions to the elemental dynamic residual into the functions of the unknown configuration only. Naturally, the above time integration should be performed at the integration points of the inertial vector $(35)_{2}$.

In linear analysis, choosing $\beta=\frac{1}{4}$ and $\gamma=\frac{1}{2}$ provides unconditional stability and second-order accuracy of the scheme. This scheme is known as the trapezoidal rule and it will be used in the numerical experiments in the next section. It is important to remember, however, that the unconditional stability in the linear regime does not extended to non-linear problems and that alternative techniques, which are designed to preserve some of the properties of motion of the actual physical system, perform better than the trapezoidal rule (Muñoz and Jelenić, in preparation).

\subsection{Newton Raphson solution procedure and update}

The virtual work Eq. (41) is tantamount to the non-linear vector equation

$$
\mathbf{g}=\mathbf{0},
$$

where $\mathbf{g}$ is the global dynamic residual of the structure. Upon introduction of a suitable interpolation for the unknown displacement and rotation fields along each element, this equation may be solved using the Newton Raphson iterative procedure. Within this procedure, the system of linear equations

$$
\mathbf{g}^{i+1}=\mathbf{g}^{i}+\mathbf{K}^{i} \Delta \mathbf{p}=\mathbf{0},
$$

where $\mathbf{K}=\nabla_{\mathbf{p}} \mathbf{g}$ is the global tangent operator and $\Delta \mathbf{p}$ is the global vector of the iterative corrections to the nodal unknowns, needs to be solved, and the nodal unknowns updated repeatedly until a converged solution is achieved. Note that the rotational field does not have to be interpolated in the same way as the virtual rotation $\delta \vartheta$ in (33). Indeed, it is not advisable that it is, since such an interpolation would lead to a loss of strain invariance in the formulation. See Jelenić and Crisfield (1999) for details of a strain-invariant interpolation for the rotational field. In the present approach, it must be borne in mind that the global residual contains the residual of the coupling element, which will give rise to additional terms in the tangent operator. Indeed, linearising the residual $\mathbf{g}_{R m}^{A}$ leads to $\Delta \mathbf{g}_{R m}=\nabla_{\mathbf{p}_{R m}} \mathbf{g}_{R m}^{A} \equiv \mathbf{K}_{c p} \Delta \mathbf{p}_{R m}$, where $\mathbf{K}_{c p}$ is the local tangent operator of the coupling element and may be expressed as

$$
\mathbf{K}_{c p}=\mathbf{N}^{\mathrm{T}} \mathbf{K}_{A} \mathbf{N}_{g}^{*}+\left[\begin{array}{c|c|c}
\mathbf{K}_{R R} & \mathbf{0}_{6 \times 6 N_{A}} & \mathbf{K}_{R m} \\
\mathbf{0}_{6 N_{A} \times 6} & \mathbf{0}_{6 N_{A} \times 6 N_{A}} & \mathbf{0}_{6 N_{A} \times 6 N_{B}} \\
\mathbf{K}_{m R} & \mathbf{0}_{6 N_{B} \times 6 N_{A}} & \mathbf{0}_{6 N_{B} \times 6 N_{B}}
\end{array}\right] .
$$

This result, including the matrices $\mathbf{N}_{g}^{*}, \mathbf{K}_{R R}, \mathbf{K}_{R m}$ and $\mathbf{K}_{m R}$, has been derived in Appendix A. Clearly, this matrix has some coupling terms between the degrees of freedom of slave element $A$ and those of the master element $B$. These terms have to be processed carefully whenever the contact point switches from an element to another element on the slideline. This is described in more detail in the following subsection.

In order to ensure that the sliding contact condition is preserved exactly, the iterative solution of the equations must include a consistent update of the kinematics. Once the iterative changes $\Delta X=\Delta \mathbf{r}_{R} \cdot \mathbf{G}_{1}$ and $\Delta \boldsymbol{\theta}_{R}$ are obtained from the solution of the system of equations (45) and the master and released variables updated, the slave kinematics $\left(\mathbf{r}_{N_{A}}, \boldsymbol{\Lambda}_{N_{A}}\right)$ at the new iteration $i+1$ is obtained according to the update process summarised in Table 1. Note that the matrix $\boldsymbol{\Lambda}\left(X_{B}^{i+1}\right)$ depends on the actual interpolation of the rotational degrees of freedom within the beam finite element used.

With all the nodal slave degrees of freedom in hand, it is now possible to update the kinematics at each integration point for every element using the adopted interpolation for the displacement and the rotation field. This in turn enables the update of the elemental contributions to the dynamic residual (35). In order to update the vector of inertial forces, one needs to perform the velocity and acceleration update described in 
Table 1

Update of slave node kinematics $\left(\mathbf{r}_{N_{A}}, \boldsymbol{\Lambda}_{N_{A}}\right)$

\begin{tabular}{llll}
\hline \multicolumn{1}{c}{$X_{B}^{i+1}$} & $X_{B}^{i}+\Delta X$ \\
\hline \multicolumn{2}{c}{ Translations } & & Rotations \\
\hline $\mathbf{r}_{N_{A}}^{i+1}$ & $\mathbf{r}\left(X_{B}^{i+1}\right)$ & $I^{j}\left(X_{B}^{i+1}\right) \mathbf{r}_{j}$ & $\boldsymbol{\Lambda}_{R}^{i+1} \exp \left(\widehat{\mathbf{T}}{ }^{1} \Delta \boldsymbol{\theta}_{R}\right) \boldsymbol{\Lambda}_{R}^{i}$ \\
& & Update $\boldsymbol{\Lambda}\left(X_{B}^{i+1}\right){ }^{i+1} \boldsymbol{\Lambda}_{\text {rel }}$ \\
\hline
\end{tabular}

the previous subsection. To update the vector of internal forces, of course, it becomes necessary to update the values of the translational and rotational strain measures.

\subsection{Contact element transition}

The present approach enables a straightforward transition of the contact point along a set of elements forming a slideline. Let us assume that at time $t_{1}$ the contact point is established between elements $A$ and $B$ (see Fig. 9), and that elements $B$ and $C$ are adjacent to each other in a string of elements on the slideline.

The contact element can be easily obtained from the value of $\mathbf{X}_{R}$ and the lengths of the elements on the slideline in the reference configuration (see Fig. 9). If the transition of the contact point between elements occurs during the iterative process, the generic definition of the coupling element allows us to consider a different contact element by just replacing $\mathbf{R}_{B}$ and $I_{B}^{j}$ in $\mathbf{g}_{R m}^{A}$ with the corresponding values for the new element $C$, i.e. $\mathbf{R}_{C}$ and $I_{C}^{j}$. Of course, special care must be exercised during the assembly of the resulting stiffness matrix since some terms couple the stiffness of the current contact element on the slideline (master element) and the sliding element $A$. When the contact point moves to another element, the topology of the coupling element still remains the same provided the new element (and, by induction, all the elements on the slideline) have the same topology. However, the vector $\mathbf{L}^{\mathrm{T}} \mathbf{g}^{N_{A}}$ in the residual and the coupling terms of $\mathbf{K}_{c p}$ will be posted into different positions in the global residual and the stiffness matrix.

Implementing this facility requires some modification to the standard data structure that should keep track of the current contact element and provide the relevant terms of the residual vector and the stiffness matrix for the coupling element. In addition, the kinematics of the current contact element must be

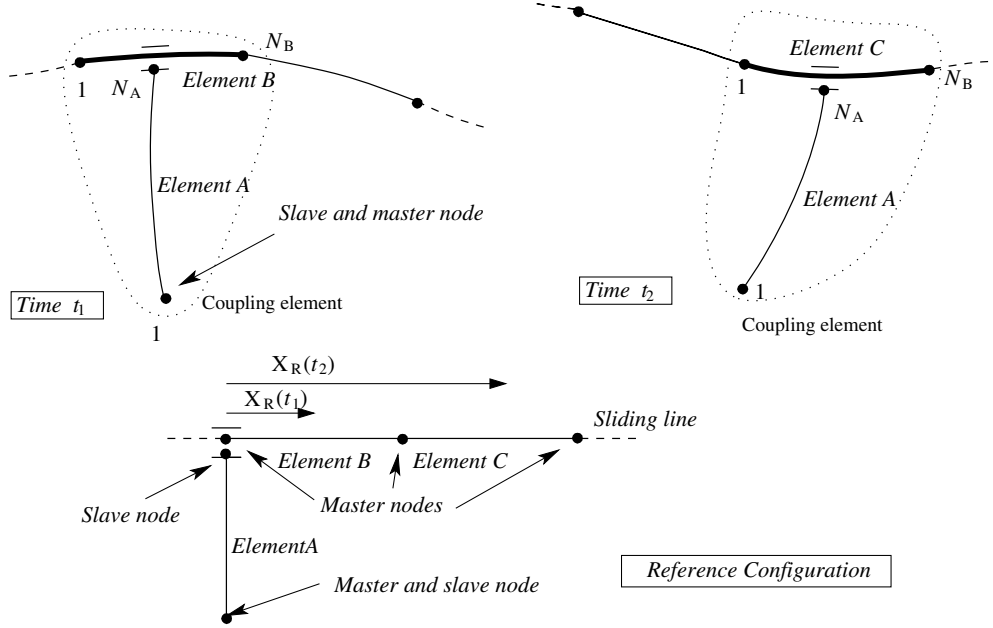

Fig. 9. Coupling element definition and scheme of the contact element transition. 


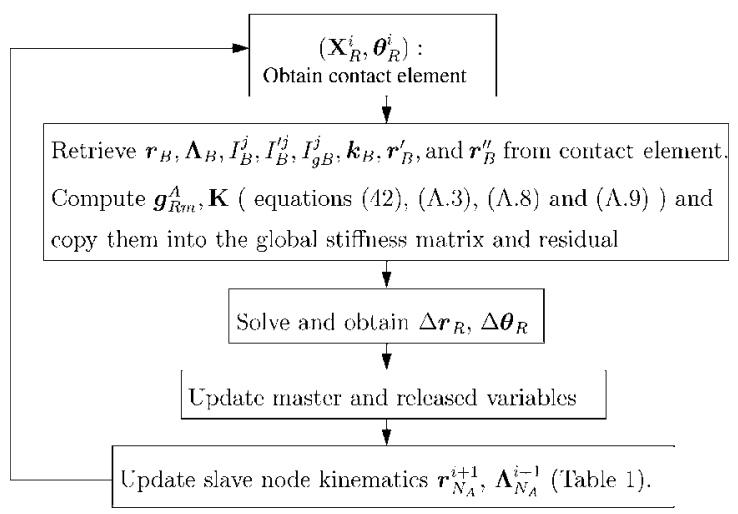

Fig. 10. Contact element update including contact transition.

retrieved in order to update the variables at the slave node $N_{A}$ (see Table 1). A scheme of the different stages required for the update and the construction of the coupling element during the iterative process is outlined in Fig. 10. The transition of the contact point between adjacent elements for the case of 3D elastodynamics can be performed accordingly.

\section{Numerical results}

\subsection{Free falling mass (Sugiyama et al., 2003)}

This example involves two flexible beams connected through a sliding joint with all the rotations released (i.e. a spherical joint attached to a sliding joint). The initial configuration of the two beams is shown in Fig. 11. The co-ordinates of the beam nodes are also given in this figure, which indicate that the beams have different lengths. All the other geometrical and material properties, however, are identical for both beams. A mass of $1 \mathrm{~kg}$ is attached to beam $B M$ at point $M$ and submitted to the gravitational field.

This example was originally run by Sugiyama et al. (2003) using their absolute nodal formulation in conjunction with a single finite element per beam. The trajectories of point $\mathrm{M}$ in the $X Z$ and $Y Z$ planes are read from Sugiyama et al. (2003) and shown in Fig. 12 using a dashed line. The authors did not give details of the time-integration scheme used.

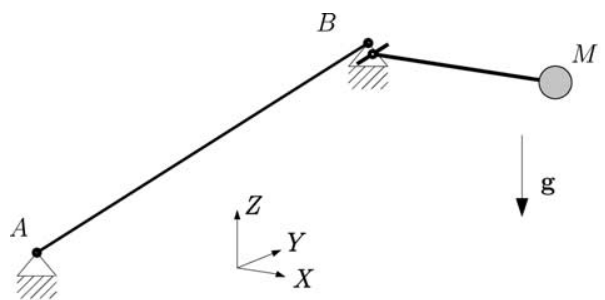

$$
\begin{aligned}
& A=\left\{\begin{array}{l}
0.0 \\
0.0 \\
0.0
\end{array}\right\} ; B=\left\{\begin{array}{l}
0.0 \\
3.0 \\
1.0
\end{array}\right\} ; M=\left\{\begin{array}{l}
1.0 \\
3.0 \\
1.0
\end{array}\right\} \\
& E I_{y y}=E I_{z z}=\frac{1}{12} \times 10^{-2} \\
& \rho I_{y y}=\rho I_{z z}=\frac{1}{12} \times 10^{-8} \\
& A E=100.0 ; \rho A=0.08 ; \nu=0.3
\end{aligned}
$$

Fig. 11. Free falling mass example. 

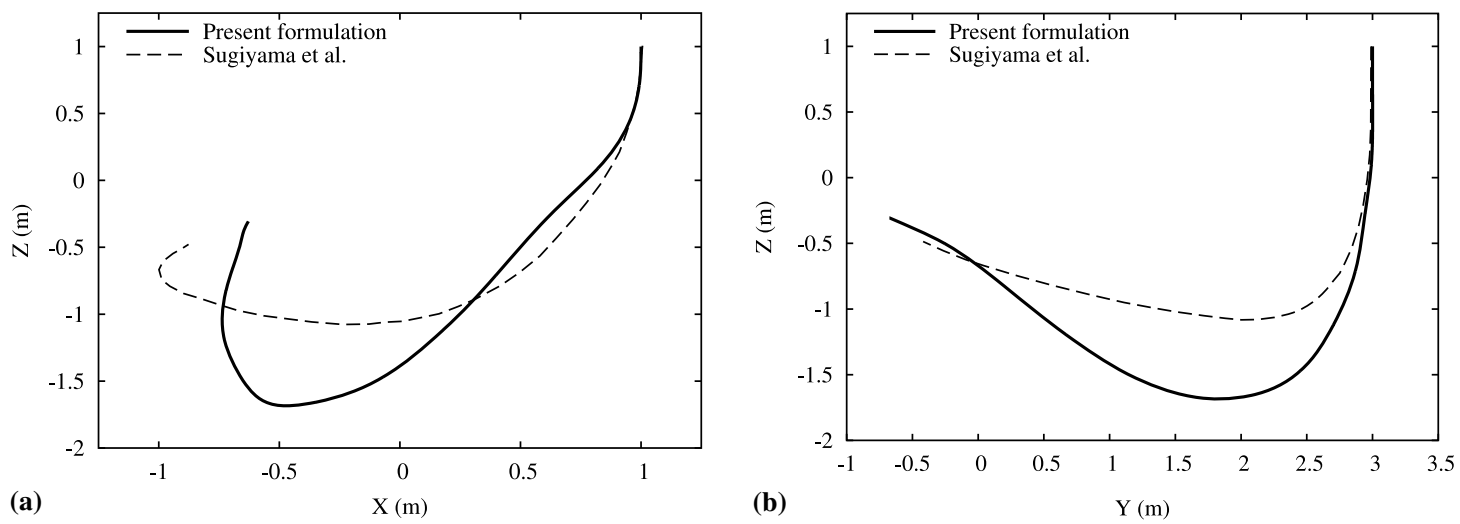

Fig. 12. Mass trajectory in the $X Z$ and $Y Z$ planes for the model given in Sugiyama et al. (2003).

Using the formulation presented in the earlier sections in conjunction with the strain-invariant geometrically exact beam elements given in Jelenić and Crisfield (1999), we have run this problem using one quadratic element per beam. The Newmark implicit scheme with $\beta=0.25$ and $\gamma=0.5$ (the trapezoidal rule) has been used for the time integration of the equations of motion with a constant time-step of magnitude $\Delta t=0.001$. The analysis has been terminated once the sliding beam reaches end $A$ of beam $A B$, which occurs at time $t=1.5911$. The projections of the trajectory of point $M$ onto co-ordinate planes $X Z$ and $Y Z$ are plotted in Fig. 12 using a solid line. Comparing these results with those obtained in Sugiyama et al. (2003) shows that while the qualitative behaviour of the structure is comparable in the two approaches, our results provide considerably larger displacements. It should be noted that the absolute nodal formulation (Sugiyama et al., 2003) uses a finite element which involves the derivatives of all the displacements at a node as the additional nodal variables, which provides a more sophisticated approximation of the axial strain. This in turn may be beneficial in systems like the present one, in which the axial straining makes a dominant contribution to the strain energy. The elements we use are, in contrast, based around the isoparametric Lagrangian interpolation of displacements and rotations as separate variables and are not expected to be competitive in problems with such a large aspect ratio between the axial and the bending strain energy. Nevertheless, this example has been chosen here in order to demonstrate the capabilites of the present formulation to deal with large displacements and a sliding joint along a single element.

\subsection{Flexible cylindrical manipulator (Krishnamurthy, 1989)}

In this example, a horizontal flexible beam with a tip mass at one of the ends is linked to a rigid hub through a sliding joint with no released rotations. The hub can rotate and move along the vertical axis as shown in Fig. 13, where the material and geometrical properties of the whole manipulator are also given. The system is subject to three time-dependent loads: force $F_{z}$, which lowers the hub, moment $M_{z}$, which rotates the hub, and follower force $F_{r}$, which pulls the flexible beam through the hub. These loads vary in time in such a manner as to move the manipulator from the position $(r, z, \theta)=(0.5588,0.5334,0)$ at $t=0$ to the position $(r, z, \theta)=(0.254,0.2286,1.5708)$ at $t=1.5$, where the degrees of freedom $r, z$ and $\theta$ are shown in Fig. 13.

This problem was solved by Krishnamurthy (1989) by considering the vibration of a beam using the engineering beam theory to be superimposed onto the three rigid-body modes. The resulting set of partial differential equations was reduced to a system of ordinary non-linear differential equations by assuming the local displacements fields for the beam to be linear combinations of the modes of vibration for a cantilever 


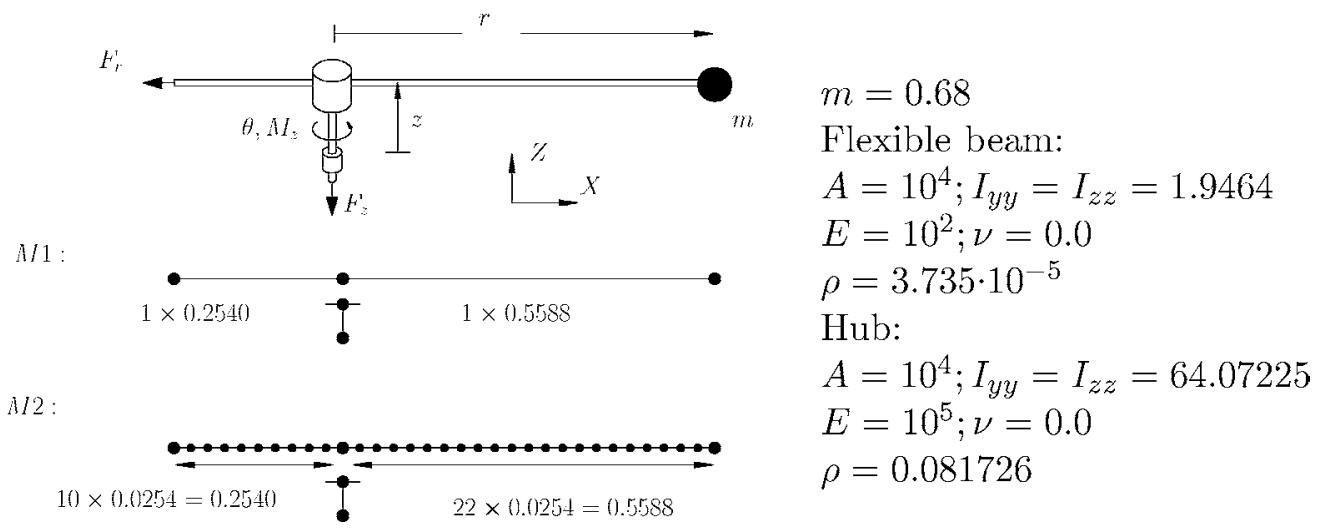

Fig. 13. Scheme and finite element models of the flexible cylindrical manipulator.

beam. No details were provided for time integration of the resulting system of differential equations. The results for the time histories of the two components of the local lateral displacement (with respect to the straight line passing through the hub opening) for both ends of the beam have been scanned from the original reference and given in Fig. 14.
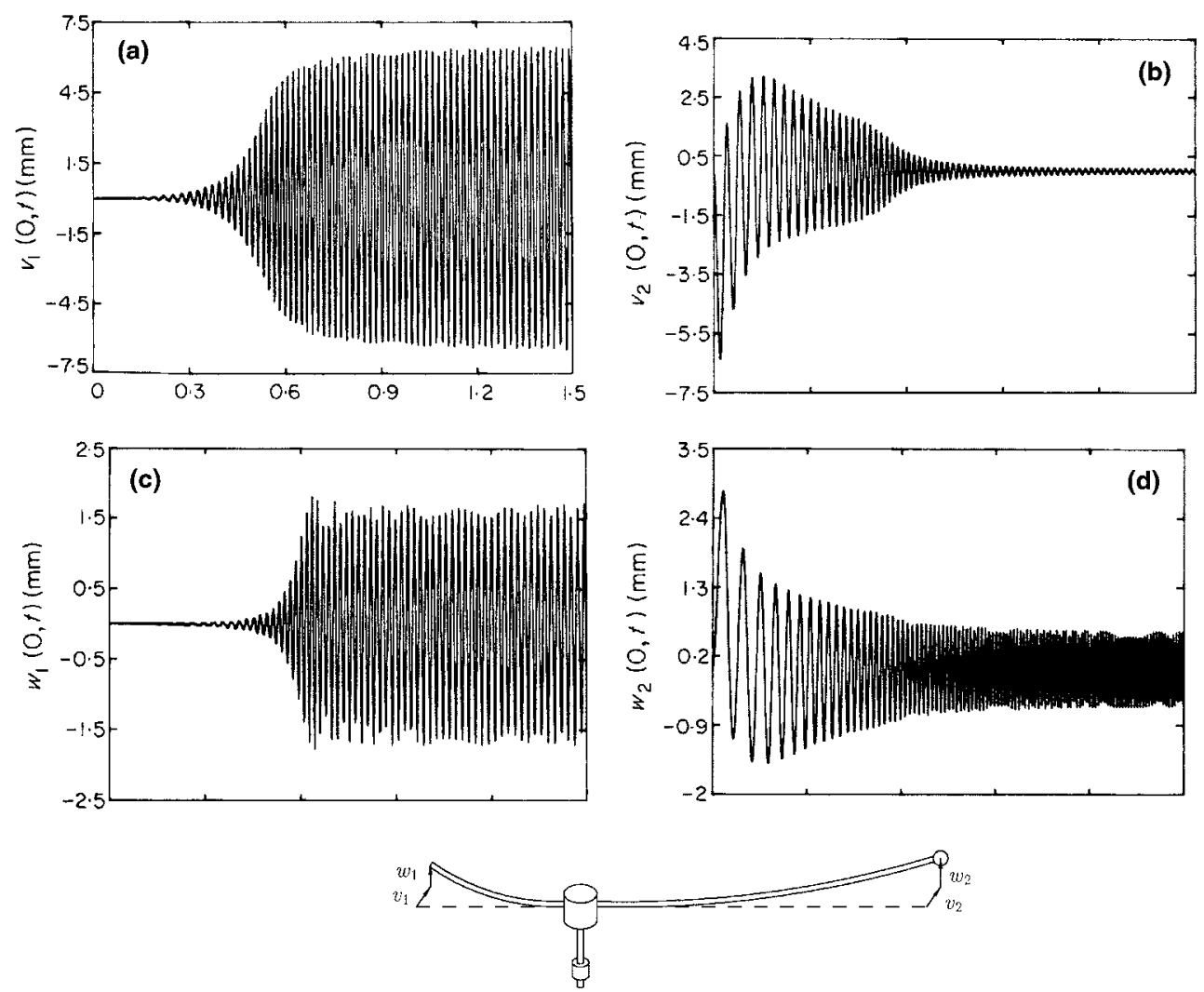

Fig. 14. Tip displacements of the manipulator given by Krishnamurthy (1989). 
In an attempt to reproduce these results using the present formulation, we have read the load histories employed by the author (Fig. 4 in Krishnamurthy (1989)). Our readings of these time histories are given in Fig. 15 (see Muñoz (2004) for the actual values). In terms of spatial discretisation, we have modelled the hub by means of a short vertical beam with equivalent mass properties and used two different finiteelement meshes to discretise the flexible beam. In the coarser mesh (mesh $M 1$ ), each cantilever end of the beam has been modelled using a single quadratic finite element whereas in the finer mesh (mesh $M 2)$, the whole beam has been modelled using 32 quadratic elements so that, in the initial configuration, one cantilever end contains 22 and the other end contains ten elements. See Fig. 13 for meshes $M 1$ and $M 2$. Invariant interpolation of total local rotations (Jelenić and Crisfield, 1999) has been used in both cases. Also, in both cases the contact between the beam and the hub is initially established at a nodal point. As the beam is being pulled through the hub, the contact point drifts away from the initial nodal point, but it always remains in contact with the beam in accordance with the presented theory. For mesh $M 1$, there is no transition of the contact point between the elements while, for mesh $M 2,15$ of the 32 elements at some point in time make contact with the hub. It is important to understand that in both models the hub is modelled as a short vertical beam, i.e. the hub is assumed to have no diameter and the sum of the lengths of the two ends of the beam makes up the total length of the beam. In reality (and in the model used in Krishnamurthy (1989)), however, the hub has certain diameter, so that the combined length of the two ends of the beam is equal to the difference between the total length of the beam and the hub diameter.
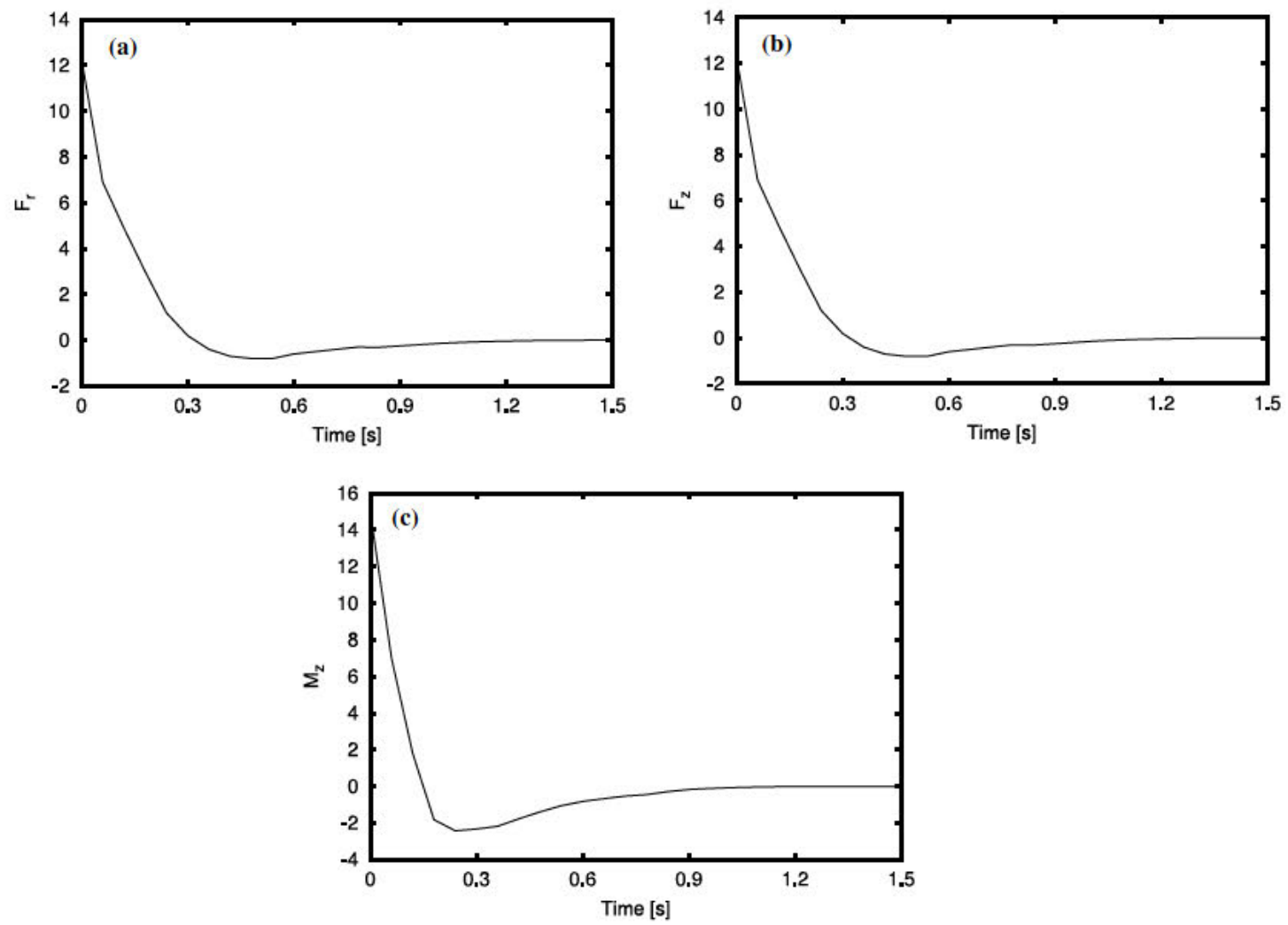

Fig. 15. Time history of the input loads $F_{r}, F_{z}$ and moment $M_{z}$. 
Both analyses have been run using the Newmark trapezoidal rule with a time-step $\Delta t=0.001$. The time step this small is required not as much for convergence reasons but rather to capture the progressively increasing frequencies of vibration of the flexible arm with the point mass as that arm becomes shorter. The resulting histories for the displacements $r$ and $z$ and the rotation $\theta$ using the two finite-element meshes are given in Fig. 16. These results are comparable to those given in Fig. 3 of Krishnamurthy (1989), but they do not correspond exactly to the expected final configuration with $(r, z, \theta)=(0.254,0.2286,1.5708)$ at $t=1.5$. It should be noted again that the loading histories in Fig. 15 where read manually from a graph in Krishnamurthy (1989).

The relative displacements $v_{1}, w_{1}, v_{2}$ and $w_{2}$ of the two ends of the flexible beam for meshes $M 1$ and $M 2$ are plotted in Figs. 17 and 18, respectively. These displacements are measured with respect to a straight line that rotates rigidly with the hub and are comparable to the original results given in Fig. 14 (Krishnamurthy, 1989). The amplitudes of $v_{1}$ and $w_{1}$ increase whereas the amplitudes and the periods of vibration of $v_{2}$ and $w_{2}$ decrease, which is what intuitively we would expect for the given loading history which tends to lengthen the arm with the tip point 1 and shorten the arm with the point mass (tip point 2). While this observation is valid for both meshes, it should be noted that the coarse mesh $M 1$ exhibits only a modest increase in the frequency of vibration of the tip mass in time (see Fig. 17).

This frequency increase is more pronounced with the finer mesh $M 2$, and it is expected that further refinement would lead to even closer agreement with the reference result (Fig. 14d). It should also be noted that this mesh manages to capture the behaviour of the vertical vibration of tip point 1 qualitatively, as can
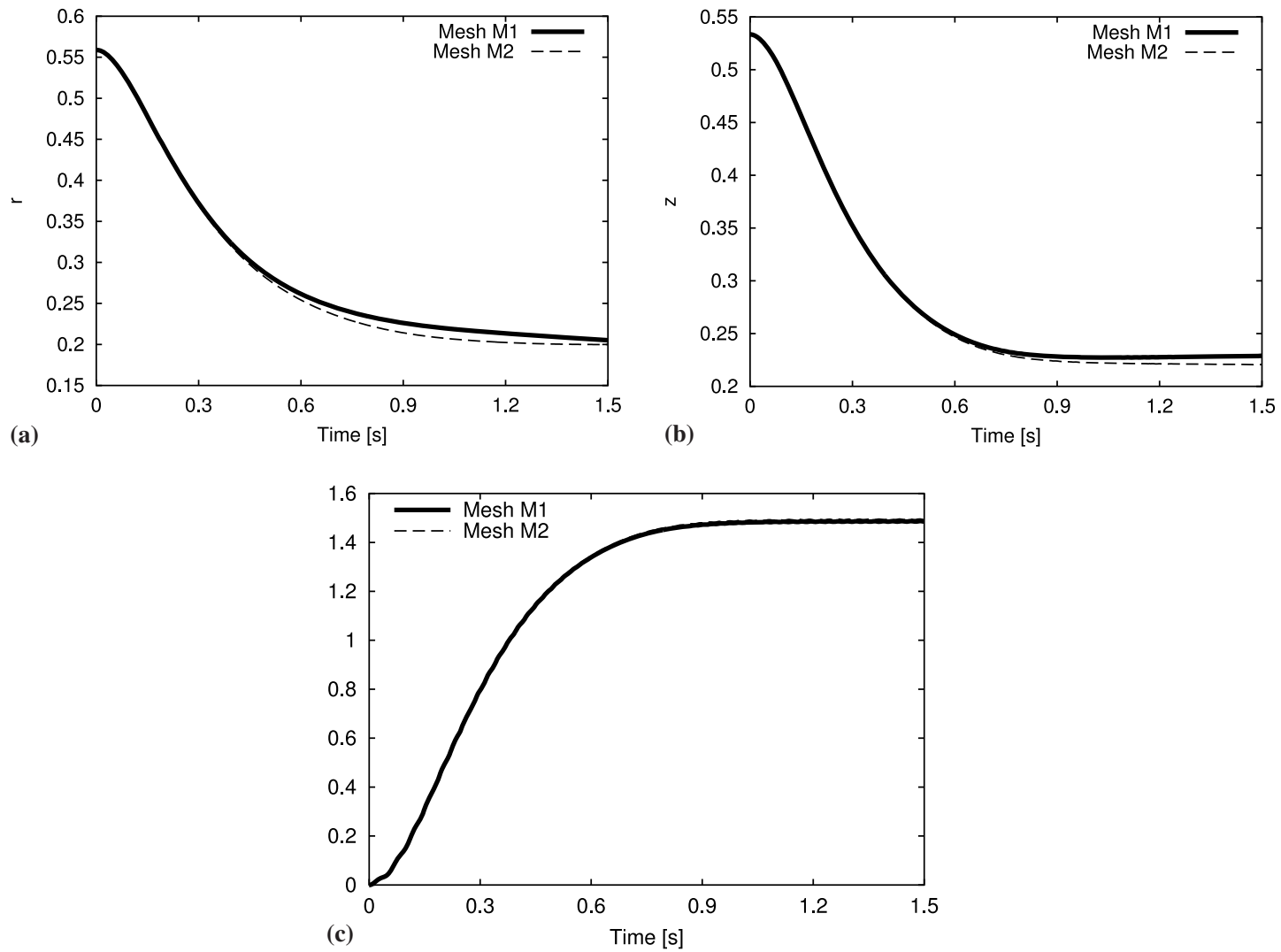

Fig. 16. Time history of the displacements $r, z$ and rotation $\theta$. 

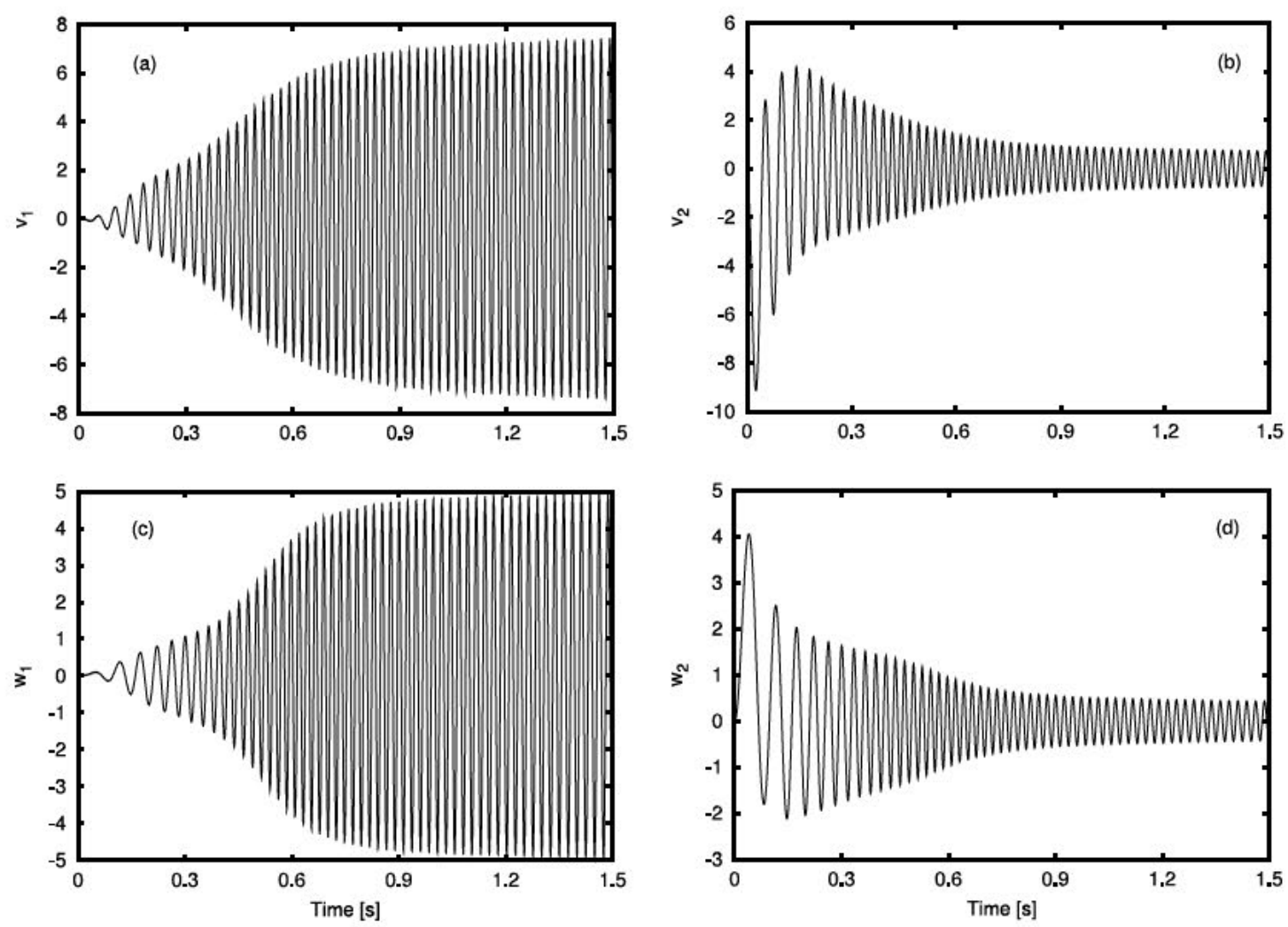

Fig. 17. Tip displacements of the flexible cylindrical manipulator using mesh $M 1$.

be observed by comparing Figs. $18 \mathrm{c}$ and $14 \mathrm{c}$. In contrast, the coarse mesh $M 1$ fails to capture this behaviour (Fig. 17c).

The differences in the displacements $v_{1}, w_{1}, v_{2}$ and $w_{2}$ between our results and the reference are in general larger for the coarse mesh $M 1$ than for the fine mesh $M 2$. Clearly, the more approximate interpolation of the contact point on the flexible arm has detrimental effects on the amplitude of vibration, especially in the vertical displacement $w_{1}$. In contrast, by modelling the flexible arm more accurately (which implies including the facility to enable the transition of the contact point between the elements), we have been able to improve the results. However, even with the finer mesh $M 2$, these amplitudes are visibly larger than those given in the reference. This difference should be attributed to the fact that in the present formulation the contact is effected at a single point. This is in contrast to the model employed in the reference, which accounts for the contact between the flexible arm and the hub along the whole diameter of the hub. As a consequence, the two ends of the arm are longer in the present model thus producing larger tip displacements.

\section{Conclusions}

A minimum-set master slave methodology for the analysis of point contacts between elastic bodies has been presented in this paper. This method does not involve Lagrangian multipliers or penalty parameters 

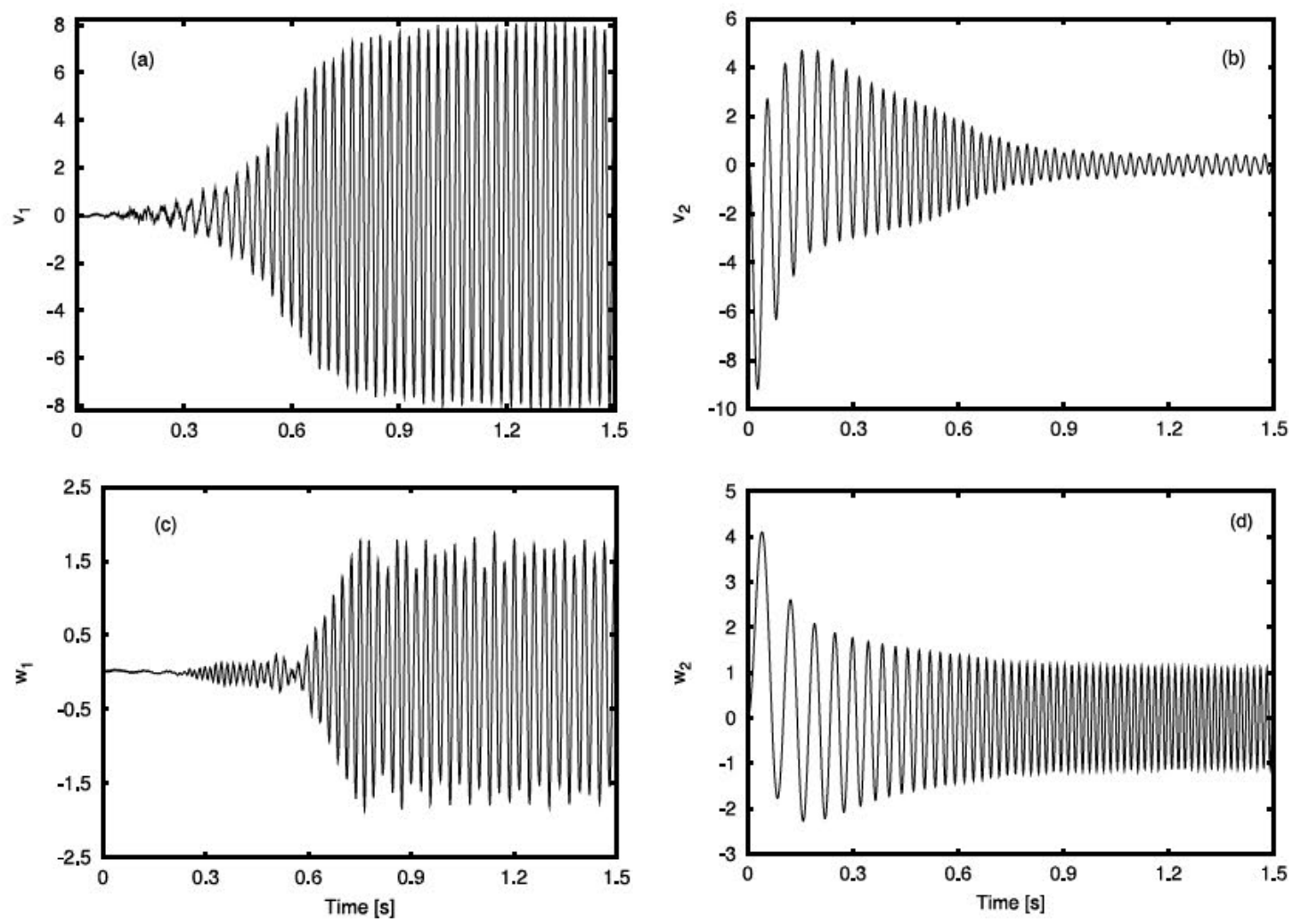

Fig. 18. Tip displacements of the flexible cylindrical manipulator using mesh $M 2$.

and may be considered as an alternative approach to addressing the contact problems in finite elastodynamics. The method has been numerically implemented in detail in the context of elastic mechanisms involving non-linear beam finite elements with joints, including the sliding joint, which is representative of the problem of contact between the elastic bodies. For such systems, the contact is retained throughout the motion, and the resulting contact force between the bodies can be of either direction.

In terms of the finite-element implementation, it is important to design the process of transition of the contact point between the adjacent elements on a slideline. It has been shown that this task can be performed by devising the so-called coupling element, which includes the degrees of freedom both of the sliding element and the (dynamically changing) contact element as well as the co-ordinate of the contact point and any additional released degrees of freedom representative of the additional joints. When the contact point jumps from one element on the slideline to another, the coupling element changes accordingly to account for the occurrence of the new contact element. The process takes a particularly simple form when all the contact elements on the slideline are of the same topology, in which case the coupling element always keeps the same number of the degrees of freedom.

The performance of the method has been presented on two numerical examples from the literature, which were originally solved using very different techniques from the present. Consequently, there have been differences between the present results and those in the references, which are in our opinion justified by the differences in the techniques used. For the free-falling mass example, a single-element mesh has been used to enable a fair comparison with the results in the reference, which were also obtained using such a 
mesh. We note, however, that these results can hardly be taken as a converged solution for such a highly elastic problem. For the flexible cylindrical manipulator, we have been able to provide a much better agreement with the reference results. This example has emphasised the need for the dynamically changing coupling element, in which case it has been possible to provide a much finer finite-element mesh, which has provided improved agreement with the reference results.

Throughout the paper, the emphasis has been placed on the rigorous definition of the contact kinematics. We believe that the performance of the method can be improved further by providing a more sophisticate time-integration technique than the presently adopted Newmark's trapezoidal rule. In particular, in a forthcoming work, we aim to investigate the performance of the time integrators which are specifically designed to preserve some of the constants of motion of the underlying system (Muñoz and Jelenić, in preparation). We also believe that the present master slave approach is worthy of further investigation in the context of general 3D contact elastodynamics.

\section{Acknowledgements}

The numerical results in this work have been obtained using the research version of the finite-element code LUSAS, kindly provided by FEA Ltd.

\section{Appendix A. Linearisation of the coupling element residual $\mathbf{g}_{R m}^{A}$}

We first note that the linearisation of the residual $\mathbf{g}_{R m}^{A}=\mathbf{N}^{\mathrm{T}} \mathbf{g}^{A}$ can be split in the following two terms:

$$
\Delta\left(\mathbf{N}^{\mathrm{T}} \mathbf{g}^{A}\right)=\mathbf{N}^{\mathrm{T}} \Delta \mathbf{g}^{A}+\left(\Delta \mathbf{N}^{\mathrm{T}}\right) \mathbf{g}^{A} .
$$

The first one can be expressed by using the elemental stiffness matrix of the sliding element $A$, denoted by $\mathbf{K}_{A}$ :

$$
\Delta \mathbf{g}^{A}=\mathbf{K}_{A} \Delta \mathbf{p}^{A} .
$$

The vector $\Delta \mathbf{p}^{A}$ can be related to the vector of iterative changes of released and master variables $\Delta \mathbf{p}_{R m}$ by using Eq. (37):

$$
\Delta \mathbf{p}^{A}=\mathbf{N}^{*} \Delta \mathbf{p}_{R m}^{A} \text {. }
$$

However, if a strain-objective interpolation is desired, local rotations and generalised shape functions $\mathbf{I}_{g B}^{j}$ for the interpolation of the iterative rotations $\Delta \boldsymbol{\vartheta}_{B}$ must be employed:

$$
\Delta \boldsymbol{\vartheta}_{B}=\mathbf{I}_{g B}^{j} \Delta \boldsymbol{\vartheta}_{j},
$$

as demonstrated in Jelenic and Crisfield (1999) where the explicit expression of the generalised shape functions can be found. Replacing the functions $I_{B}^{j}$ in matrix $\mathbf{N}^{*}$ by the strain-invariant shape functions $\mathbf{I}_{g B}^{j}$, we obtain the transformation matrix $\mathbf{N}_{g}^{*}$ relating the vectors $\Delta \mathbf{p}^{A}$ and $\Delta \mathbf{p}_{R m}^{A}$ :

$$
\Delta \mathbf{p}^{A}=\mathbf{N}_{g}^{*} \Delta \mathbf{p}_{R m}^{A}
$$

with

$$
\mathbf{N}_{g}^{*}=\left[\begin{array}{cccccc}
\mathbf{0} & \mathbf{I} & \ldots & \mathbf{0} & \mathbf{0} & \mathbf{0}_{6 \times 6 N_{B}} \\
\vdots & \vdots & \ddots & \vdots & \vdots & \vdots \\
\mathbf{0} & \mathbf{0} & \ldots & \mathbf{I} & \mathbf{0} & \mathbf{0}_{6 \times 6 N_{B}} \\
\mathbf{R}_{B}^{*} & \mathbf{0} & \ldots & \mathbf{0} & \mathbf{0} & \mathbf{L}_{g}
\end{array}\right] ; \quad \mathbf{L}_{g}=\left[\begin{array}{ccccc}
I_{B}^{1} \mathbf{I} & \mathbf{0} & \ldots & I_{B}^{N_{B}} \mathbf{I} & \mathbf{0} \\
\mathbf{0} & \mathbf{I}_{g B}^{1} & \ldots & \mathbf{0} & \mathbf{I}_{g B}^{N_{B}}
\end{array}\right]
$$


Thus, making use of the elemental stiffness matrix $\mathbf{K}_{A}$ and matrix $\mathbf{N}_{g}^{*}$, the term $\Delta \mathbf{g}^{A}$ in (A.1) reads

$$
\Delta \mathbf{g}^{A}=\mathbf{K}_{A} \Delta \mathbf{p}_{s}=\mathbf{K}_{A} \mathbf{N}_{g}^{*} \Delta \mathbf{p}_{R m} .
$$

In order to obtain an explicit form for the second term in (A.1) with $\mathbf{N}$ given in (42) the following preliminary results are deduced:

$$
\begin{aligned}
& \Delta \mathbf{r}_{B}^{\prime}=\Delta\left(I_{B}^{\prime j} \mathbf{r}_{j}\right)=I_{B}^{\prime j} \Delta \mathbf{r}_{j}+I_{B}^{\prime \prime j} \mathbf{r}_{j} \Delta X_{C}=I_{B}^{\prime j} \Delta \mathbf{r}_{j}+\mathbf{r}_{B}^{\prime \prime} \otimes \mathbf{G}_{1} \Delta \mathbf{r}_{R}, \\
& \Delta \boldsymbol{\Lambda}_{B}=\widehat{\Delta \vartheta} \boldsymbol{\Lambda}_{B}+\Delta X_{B} \widehat{\mathbf{k}}_{B} \boldsymbol{\Lambda}_{B}=\left(\widehat{\Delta \vartheta}+\left(\Delta \mathbf{r}_{R} \cdot \mathbf{G}_{1}\right) \widehat{\mathbf{k}}_{B}\right) \boldsymbol{\Lambda}_{B}
\end{aligned}
$$

where $\mathbf{k}_{B}=\boldsymbol{\Lambda}_{B} \boldsymbol{\kappa}_{B}$ is the (spatial) curvature.

The linearisation of the transformation matrix $\mathbf{T}^{-\mathrm{T}}$ can be written as (see Ritto-Corrêa and Camotim, 2002)

$$
\Delta\left(\mathbf{T}(\boldsymbol{\theta})^{-\mathrm{T}}\right) \mathbf{a}=\mathbf{D}(\boldsymbol{\theta}, \mathbf{a}) \Delta \boldsymbol{\theta}
$$

where the matrix $\mathbf{D}(\boldsymbol{\theta}, \mathbf{a})$ is given by

$$
\mathbf{D}(\boldsymbol{\theta}, \mathbf{a})=c_{1} \widehat{\mathbf{a}}+c_{2}(\widehat{\mathbf{a}} \widehat{\boldsymbol{\theta}}-2 \widehat{\boldsymbol{\theta}} \widehat{\mathbf{a}})-c_{3} \widehat{\boldsymbol{\theta}} \mathbf{a} \otimes \boldsymbol{\theta}+c_{4} \widehat{\boldsymbol{\theta}}^{2} \mathbf{a} \otimes \boldsymbol{\theta}
$$

and

$$
c_{1}=\frac{1-\cos \theta}{\theta^{2}} \quad c_{2}=\frac{1}{\theta^{2}}\left(1-\frac{\sin \theta}{\theta}\right) \quad c_{3}=\frac{\theta \sin \theta-2(1-\cos \theta)}{\theta^{4}} \quad c_{4}=\frac{3 \sin \theta-\theta(2+\cos \theta)}{\theta^{5}} .
$$

Making use of (A.5) and (A.6), the computation of $\left(\Delta \mathbf{N}^{\mathrm{T}}\right) \mathbf{g}^{A}$ becomes

$$
\begin{aligned}
& \left(\Delta \mathbf{N}^{\mathrm{T}}\right) \mathbf{g}^{A}=\left\{\begin{array}{c}
\mathbf{G}_{1} \otimes \Delta \mathbf{r}_{B_{1}}^{\prime} \mathbf{g}_{n}^{N_{A}} \\
\Delta\left(\mathbf{T}_{R}^{-\mathrm{T}} \boldsymbol{\Lambda}_{B_{1}}^{\mathrm{T}}\right) \mathbf{g}_{m}^{N_{A}} \\
\mathbf{0}_{6 N_{A}} \\
\Delta\left(I_{B}^{1}\right) \mathbf{g}^{N_{A}} \\
\vdots \\
\Delta\left(I_{B}^{N_{B}}\right) \mathbf{g}^{N_{A}}
\end{array}\right\}=\left\{\begin{array}{c}
\mathbf{G}_{1} \otimes \mathbf{g}_{n}^{N_{A}}\left(I_{B}^{j \prime} \Delta \mathbf{r}_{j}+\mathbf{r}_{B}^{\prime \prime} \otimes \mathbf{G}_{1} \Delta \mathbf{r}_{R}\right) \\
\mathbf{D}\left(\boldsymbol{\theta}_{R}, \boldsymbol{\Lambda}_{B}^{\mathrm{T}} \mathbf{g}_{m}^{N_{A}}\right) \Delta \boldsymbol{\theta}_{R}-\mathbf{T}_{R}^{-\mathrm{T}} \boldsymbol{\Lambda}_{B}^{\mathrm{T}} \widehat{\mathbf{k g}}_{m}^{N_{A}} \Delta X_{B}+\mathbf{T}_{R}^{-\mathrm{T}} \boldsymbol{\Lambda}_{B}^{\mathrm{T}} \widehat{\mathbf{g}}_{m}^{N_{A}} \Delta \boldsymbol{\vartheta}_{B} \\
\mathbf{0}_{6 N_{A}} \\
I_{B}^{1 !} \mathbf{g}^{N_{A}} \Delta X_{B} \\
\vdots \\
I_{B}^{N_{A}} \mathbf{g}^{N_{A}} \Delta X_{B}
\end{array}\right\} \\
& =\left[\begin{array}{c|c|c}
\mathbf{K}_{R R} & \mathbf{0}_{6 \times 6 N_{A}} & \mathbf{K}_{R m} \\
\mathbf{0}_{6 N_{A} \times 6} & \mathbf{0}_{6 N_{A} \times 6 N_{A}} & \mathbf{0}_{6 N_{A} \times 6 N_{B}} \\
\mathbf{K}_{m R} & \mathbf{0}_{6 N_{B} \times 6 N_{A}} & \mathbf{0}_{6 N_{B} \times 6 N_{B}}
\end{array}\right] \Delta \mathbf{p}_{R m},
\end{aligned}
$$

where

$$
\begin{aligned}
\mathbf{K}_{R R}= & {\left[\begin{array}{cc}
\mathbf{r}_{B}^{\prime \prime} \cdot \mathbf{g}_{n}^{N_{A}} \mathbf{G}_{1} \otimes \mathbf{G}_{1} & \mathbf{0} \\
\mathbf{T}_{R}^{-\mathrm{T}} \boldsymbol{\Lambda}_{B}^{\mathrm{T}} \widehat{\mathbf{g}}_{m}^{N_{A}} \mathbf{k}_{B} \otimes \mathbf{G}_{1} & \mathbf{D}\left(\boldsymbol{\theta}_{R}, \boldsymbol{\Lambda}_{B}^{\mathrm{T}} \mathbf{g}_{m}^{N_{A}}\right)
\end{array}\right], } \\
\mathbf{K}_{R m}= & {\left[\begin{array}{cc}
\mathbf{G}_{1} \otimes \mathbf{g}_{n}^{N_{A}} & \mathbf{0} \\
\mathbf{0} & \mathbf{T}_{R}^{-\mathrm{T}} \boldsymbol{\Lambda}_{B}^{\mathrm{T}} \widehat{\mathbf{g}}_{m}^{N_{A}}
\end{array}\right]\left[\begin{array}{ccccc}
I_{B}^{1 \prime} \mathbf{I} & \mathbf{0} & \ldots & I_{B}^{N_{B} \prime} \mathbf{I} & \mathbf{0} \\
\mathbf{0} & \mathbf{I}_{g B}^{1} & \ldots & \mathbf{0} & \mathbf{I}_{g B}^{N_{B}}
\end{array}\right], } \\
\mathbf{K}_{m R}= & {\left[\begin{array}{c}
I_{B}^{1 / \mathbf{I}} \\
\vdots \\
I_{B}^{N_{A} \prime \mathbf{I}}
\end{array}\right] \mathbf{g}^{N_{A}} \otimes \mathbf{G}_{1} . }
\end{aligned}
$$


Eventually, the total stiffness matrix of the coupling element $\mathbf{K}_{c p}$ can thus be written as

$$
\mathbf{K}_{c p}=\mathbf{N}^{\mathrm{T}} \mathbf{K}_{A} \mathbf{N}_{g}^{*}+\left[\begin{array}{c|c|c}
\mathbf{K}_{R R} & \mathbf{0}_{6 \times 6 N_{A}} & \mathbf{K}_{R m} \\
\mathbf{0}_{6 N_{A} \times 6} & \mathbf{0}_{6 N_{A} \times 6 N_{A}} & \mathbf{0}_{6 N_{A} \times 6 N_{B}} \\
\mathbf{K}_{m R} & \mathbf{0}_{6 N_{B} \times 6 N_{A}} & \mathbf{0}_{6 N_{B} \times 6 N_{B}}
\end{array}\right] .
$$

\section{References}

Argyris, J., 1982. An excursion into large rotations. Comput. Methods Appl. Mech. Engng. 32, 81155.

Bauchau, O., 2000. On the modeling of prismatic joints in flexible multi body systems. Comput. Methods Appl. Mech. Engng. 181, 87 105.

Bauchau, O., Bottasso, C., 2001. Contact conditions for cylindrical, prismatic, and screw joints in flexible multibody systems. Multibody Syst. Dynam. 5, 251278.

Cardona, A., Géradin, M., 1988. A beam finite element non linear theory with finite rotations. Int. J. Numer. Methods Engng. 26, 24032438 .

Crisfield, M., 1997. Non Linear Finite Element Analysis of Solids and Structures Advanced Topics, vol. 2. John Wiley \& Sons, Chichester.

Crisfield, M., Jelenić, G., 1998. Finite element analysis and deployable structures. In: Deployable Structures: Theory and Applications. IUTAM, Cambridge, pp. 8796.

Crisfield, M., Jelenić, G., 2000. Energy/momentum conserving time integration procedures with finite elements and large rotations. In: Ambrósio, J., Kleiber, M. (Eds.), Computational Aspects of Nonlinear Structural Systems with Large Rigid Body Motion. IOS Press, Pultusk, Poland, pp. 181200.

García de Jalón, J., Bayo, E., 1994. Kinematic and Dynamic Simulation of Multibody Systems The Real Time Challenge. Springer Verlag, New York.

Géradin, M., Cardona, A., 2001. Flexible Multibody Dynamics. A Finite Element Approach. John Wiley \& Sons.

Ibrahimbegović, A., Mamouri, S., 2000. On rigid components and joint constraints in nonlinear dynamics of flexible multibody systems employing 3D geometrically exact beam model. Comput. Methods Appl. Mech. Engng. 188, 805831.

Jelenić, G., Crisfield, M., 1996. Non linear master slave relationships for joints in 3D beams with large rotations. Comput. Methods Appl. Mech. Engng. 135, 211228.

Jelenić, G., Crisfield, M., 1999. Geometrically exact 3D beam theory: implementation of a strain invariant finite element for statics and dynamics. Comput. Methods Appl. Mech. Engng. 171, 141171.

Jelenić, G., Crisfield, M., 2001. Dynamic analysis of 3D beams with joints in presence of large rotations. Comput. Methods Appl. Mech. Engng. 32 33, 41954230.

Krishnamurthy, K., 1989. Dynamic modelling of a flexible cylindrical manipulator. J. Sound Vib. 132 (1), 143154.

Marjamaki, H., Makinen, J., 2003. Modelling telescopic boom the plane case: Part I. Comput. Struct. 81, 15971609.

Marsden, J., Hughes, T., 1994. Mathematical Foundations of Elasticity. Dover Publications.

Mitsugi, J., 1997. Direct strain measure for large displacement analyses on hinge connected beam structures. Comput. Struct. 64, 509 517.

Munoz, J., 2004. Finite element analysis of flexible mechanisms using the master slave approach with emphasis on the modelling of joints. Ph.D. thesis, Imperial College London.

Munoz, J., Jelenic, G., in preparation. Conserving time integration schemes for flexible mechanisms with sliding joints.

Munoz, J., Jelenic, G., Crisfield, M., 2003. Master slave approach for the modelling of joints with dependent degrees of freedom in flexible mechanisms. Commun. Numer. Methods Engng. 19, 689702.

Newmark, N., 1959. A method of computation for structural dynamics. J. Engin. Mech. Div. 85 (EM3), 6794.

Ogden, R., 1984. Non Linear Elastic Deformations, vol. 26 Dover Publications.

Reissner, E., 1972. On one dimensional finite strain beam theory: the plane problem. J. Appl. Math. Phys. (ZAMP) 23, 795804.

Ritto Corrêa, M., Camotim, D., 2002. On the differentiation of Rodrigues formula and its significance for the vector like parametrization of Reissner Simo beam theory. Int. J. Numer. Methods Engng. 55 (9), 10051032.

Simo, J., 1985. A finite strain beam formulation. The three dimensional dynamics problem. Part I. Comput. Methods Appl. Mech. Engng. 49, 5570.

Simo, J., Vu Quoc, L., 1986. A three dimensional finite strain rod model. Part II: Computational aspects. Comput. Methods Appl. Mech. Engng. 58, 79116. 
Simo, J., Wong, K., 1991. Unconditionally stable algorithms for rigid body dynamics that exactly preserve energy and momentum. Int. J. Numer. Methods Engng. 31, 1952.

Sugiyama, H., Escalona, J., Shabana, A., 2003. Formulation of three dimensional joint constraints using the absolute nodal coordinates. Nonlin. Dynam. 31, 167195.

von Schwerin, R., 1999. Multibody Systems Simulation. Numerical Methods, Algorithms and Software. Springer Verlag, New York. 